\title{
Tuning PID controllers with Symmetric Send-on-Delta sampling strategy
}

\author{
Oscar Miguel-Escrig*, Julio-Ariel Romero-Pérez, Roberto Sanchis-Llopis \\ Department of System Engineering and Design. Universitat Jaume I. \\ Campus del Riu Sec Avda. Vicent Sos Baynat s/n 12071. \\ Castelló de la Plana. Spain.
}

\begin{abstract}
A procedure for tuning PID controllers with SSOD sampling for FOPTD systems is proposed. It is based on the definition of a new robustness measure to avoid limit cycle oscillations, called the Tsypkin margin $\left(M_{T}\right)$. This margin is based on the Tsypkin method and does not rely on the attenuation of high order harmonics, as the describing function approaches require. Therefore, the avoidance of limit cycle oscillations can be guaranteed for any system, as a difference with the describing function based procedures. The procedure allows to obtain the PID controller that minimizes the disturbance IAE while fulfilling constraints on robustness to oscillations and on control action bumps due to the SSOD sampling. A freely available Java tool has been developed in order to simplify the application of the tuning procedure. In case of a non FOPTD system, it first calculates an approximate FOPTD model. The paper shows that the derivative filter parameter, $N$ is a critical tuning parameter in order to find a compromise between performance and control action bumps.
\end{abstract}

Keywords: event-based-PID; FOPTD; SSOD; tuning procedure; robustness 2010 MSC: 00-01, 99-00

\section{Introduction}

Nowadays event based controllers are a promising alternative to the classical time driven control systems to reduce the measurement frequency needed for the control without degrading the closed loop performance. This is a basic requirement for controllers in networked control systems where many devices (sensors, actuators, controllers) share a communication channel with limited bandwidth. The reduction in the number of transmitted messages improve the network overall behavior, for example avoiding dropouts and delays. The use of wireless communications in control applications has also encouraged the development of event based controllers. In this case, the reduction of data transmission implies an important decrease in power consumption, therefore increasing the lifetime of batteries of self-powered remote sensors [9].

The reduction of the measurement frequency in event based control systems strongly depends on the strategy used to generate the events to send data through the network. In that sense, send-on-delta (SOD) strategy has emerged as one of the most promising approaches, which reduces considerably the sending of new data $[8,15]$. The SOD method consists in transmitting data from the sensor to the controller node only if the measurement value changes more than a given specified $\delta$ value, [13]. A particular kind of SOD, named symetric-send-on-delta (SSOD), is proposed in [5]. The SSOD sampler quantifies the input signal by a quantity multiple of a constant value $\delta$, then, a new value of the input is sent by the sampler when the input changes in a quantity $\delta$.

One of the key points in the analysis and design of event-based control systems is the existence of limit cycles that can lead to sustained oscillations in the closed loop response. The characterization of limit cycles for different kind of systems, such as integrator processes plus time delay (IPTD), first order processes plus time delay (FOPTD), and second-order processes plus time delay (SOPTD), when using a SSOD sampling strategy has been presented in [6]. The study of the limit cycles is important to predict the magnitude and frequency of the oscillation that

\footnotetext{
${ }^{*}$ Corresponding author

Email addresses: omiguel@uji.es (Oscar Miguel-Escrig), romeroj@uji.es (Julio-Ariel Romero-Pérez), rsanchis@uji.es (Roberto Sanchis-Llopis)
} 
could appear in a system as a consequence of the detuning of the controller produced by changes in the system's dynamic. From the PID tuning point of view, one of the main goal is to prevent limit cycles in order to avoid undesired oscillations which can reduce the overall performance of the control system or produce excessive actuator wear.

In [16] and [14], tuning methods for PI controllers with SSOD sampler have been developed based in new robustness margins for limit cycles, that were obtained by applying the describing function (DF) technique and entail with the classical concepts of phase and gain margins. The same approach was used in [18], where a unified design of SSOD-PID control architecture for self-regulating and integral processes was investigated. The use of the DF let to introduce tools of the classical control theory, such as the Nyquist plot, for the analysis and design of event based control systems. That could make the design of event based PID controllers more understandable and consequently boost its application in industry, since these concepts are generally taught in the automatic control undergraduate courses.

It is well known, however, that the validity of the DF depends on the filtering properties of the open loop transfer function: only under certain filtering conditions the higher order harmonics can be neglected and the DF can be successfully applied. Therefore, low order models, such FOPTD and SOPTD, which are commonly used to describe actual industrial processes, are excluded from this approach and new methods are required to use frequency response based strategies. Concerning the kind of controller, the use of PID which increase the bandwidth respect to the PI case could be an additional cumbersome for the application of the DF. In [12], the authors proposed a new robustness measure to avoid limit cycle on SSOD based PI controllers. The proposal is based on the Tsypkin's method [20], which has been widely used to study the relay control systems, that is the reason why we named the robustness measure Tsypkin's margin $\left(M_{T}\right)$. Unlike the margins proposed in [16] and [14], which are based on the DF, the robustness margin $M_{T}$ is valid not only for systems with good filtering capabilities but also for systems whose dynamic is described by low order models.

In this paper, we propose a new method for tuning PID controllers for FOPTD systems using a SSOD sampling strategy. It is well known that the FOPTD models admit a dimensionless representation that allows to express some results, including homogeneous tuning rules that depend on the quotient between the time delay and time constant, [3]. We combine this idea with the concept of Tsypkin's margin to develop a general framework for tuning SSOD based PID controllers. The proposal can be applied to any homogeneous tuning rule in order to evaluate its performance when applied to SSOD based PID. This general framework has been then applied to the tuning method developed by the author in [19] resulting in a set tuning rules for SSOD-PID which takes into account an optimum trade-off between IAE index, the control action variations due to changes of magnitude $\delta$ on the measured variable, and the robustness to limit cycle expressed in terms of $M_{T}$.

The tuning of SSOD based PI controllers for FOPTD systems has been previously addressed in [4], where some rules were designed by minimizing the $1 \%$ settling time of the closed loop response. Our study take into account both PI and PID structures, and it proves that the latter can significantly improve the system performance without degrading the robustness to limit cycles measured in terms of $M_{T}$. Additionally, an extensive study about the effect of the derivative filter coefficient on the closed loop behavior is also presented here.

\section{Problem statement}

Consider the networked control system shown in Figure 1, where $C(s)$ and $G(s)$ are the controller and the process transfer functions respectively, $y_{r}$ is the reference signal to be tracked, $y$ is the controlled output, and $p$ is the disturbance input. It is supposed that the controller is located near the actuator and the sensor sends measurements of process output $y$ (or more precisely of the tracking error $e$ ) to the controller through a communication network using the SSOD strategy. The ZOH block keeps in $\bar{e}$ the last sent value of process output $e^{*}$ until a new value is transmitted by the SSOD block. Communication delays through the network are represented by the term $\exp \left(-t_{d} s\right)$.

This control scheme was first proposed in [5] considering $C(s)$ a PI controller, so the authors called it SSOD-PI architecture. As commented in the introduction, some guide lines for PI tuning were given in [4] when the process $G(s)$ is a FOPTD model. Our goal in this paper is to design a PID controller with transfer function given by equation (1) in order to reach an adequate performance and robustness of the closed-loop system. The objective of the tuning procedure is not only to guarantee stability but also to avoid persistent oscillations or limit cycles that can appear due to the SSOD strategy if the controller is not designed properly. 


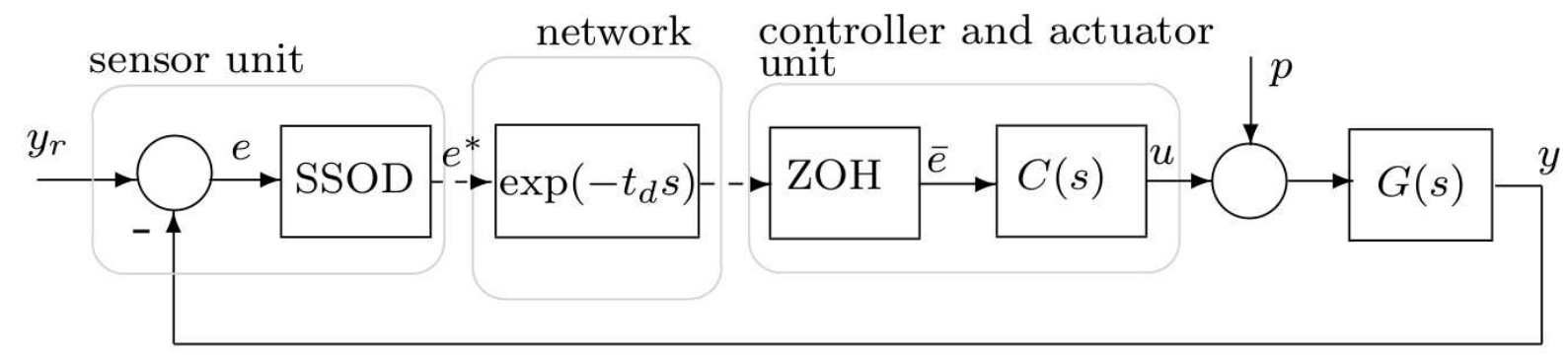

Figure 1: Networked control system with SSOD sampling strategy. SSOD- $C(s)$ architecture.

$$
C(s)=K_{p}\left(1+\frac{1}{T_{i} s}+\frac{N T_{d} s}{T_{d} s+N}\right)
$$

Previous works have addressed the tuning of SSOD-PI controllers by predicting limit cycles using the Describing Function [16, 14], a well known tool used in the analysis of non-linear control systems. In those works one of the design objectives was to avoid the intersection between the negative inverse of the DF with the open-loop transfer function on the polar plot. Nevertheless, it is known that the DF technique relies on the filtering capabilities of the linear part of the control loop, which must be capable of filter enough the high frequency harmonics. The linear part involves both the process and the controller dynamics, therefore, for processes with low filtering capabilities and PID controllers, which trend to increase the bandwidth respect to the PI case, the DF could not be a suitable approach to analyze the existence of limit cycles. This fact is illustrated through the following examples.

Example 1. Consider the FOPTD model whose transfer function is:

$$
G(s)=\frac{e^{-0.2 s}}{s+1}
$$

Controllers $C(s)$ have been tuned according to Ziegler-Nichols [21], Cohen-Coon [7], AMIGO [1] and SanchisRomero-Balaguer [19] (SRB for the sake of brevity) methods. The resulting PID parameters are gathered in Table 1. The system in Figure 1 admits the Hammerstein-Wiener representation shown in Figure 2, being SSOD_ZOH the combination of SSOD and $\mathrm{ZOH}$ blocks. The condition to avoid limit cycles is,

$$
G_{o l}(j \omega) \neq-\frac{1}{\mathcal{N}}, \quad \forall \omega
$$

where $G_{o l}(j \omega)$ is the open-loop transfer function:

$$
G_{o l}(j \omega)=\exp \left(-t_{d} j \omega\right) C(j \omega) G(j \omega)
$$

and $\mathcal{N}$ is the describing function of the SSOD_ZOH block. Graphically this condition implies no intersection between the Nyquist diagram and the negative inverse of $\mathcal{N}$.

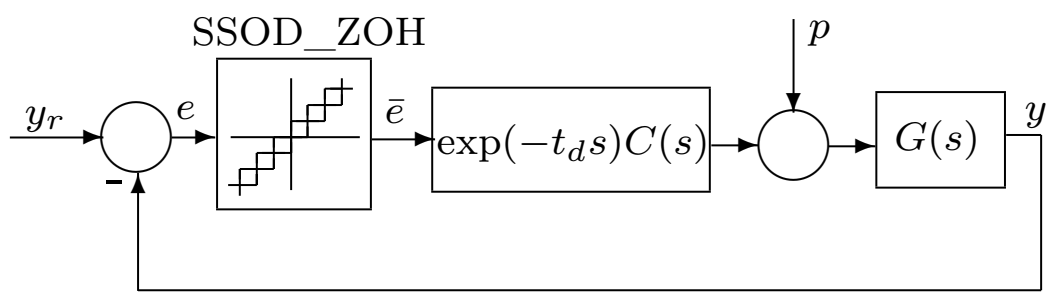

Figure 2: Non-linear equivalent system to the control system with SSOD sampling strategy in Figure 1.

Figure 3 shows the Nyquist diagrams obtained with the controllers in Table 1 and the plot of $-1 / \mathcal{N}$. It can be seen that the open-loop transfer functions corresponding to Ziegler-Nichols and Cohen-Coon methods intersect 
Table 1: PID Parameters with different tuning rules for the system presented in equation (2).

\begin{tabular}{c|c|c|c|c} 
& $\mathbf{K}_{\mathbf{p}}$ & $\mathbf{T}_{\mathbf{i}}$ & $\mathbf{T}_{\mathbf{d}}$ & $\mathbf{N}$ \\
\hline Ziegler-Nichols & 6 & 0.4 & 0.1 & 10 \\
Cohen-Coon & 6.917 & 0.455 & 0.07 & 10 \\
AMIGO & 2.45 & 0.587 & 0.094 & 10 \\
SRB & 2.181 & 0.484 & 0.115 & 10
\end{tabular}

with $-1 / \mathcal{N}$, so these systems will present limit cycle oscillations. This is not the case for AMIGO and SRB methods, for which intersections between $G_{o l}$ and $-1 / \mathcal{N}$ do not take place, consequently, they are not expected to oscillate. Simulations of the controlled systems presented in Figure 4 refute the predictions based on the DF since the four controllers lead to oscillatory behaviors. As it can be seen each controller induces oscillations with different waveforms because the difference in the tuning parameters produce different $G_{\text {ol }}(s)$, each one with its own limit cycle. Additionally, it is worth noticing that the waveforms in Figure 4 are, generally speaking, hardly sinusoidal. This is due to the failure of $G_{o l}(s)$ to attenuate higher harmonics sufficiently. It is the waveform's harmonic content which is responsible for the unsuccessful application of the DF in this example.

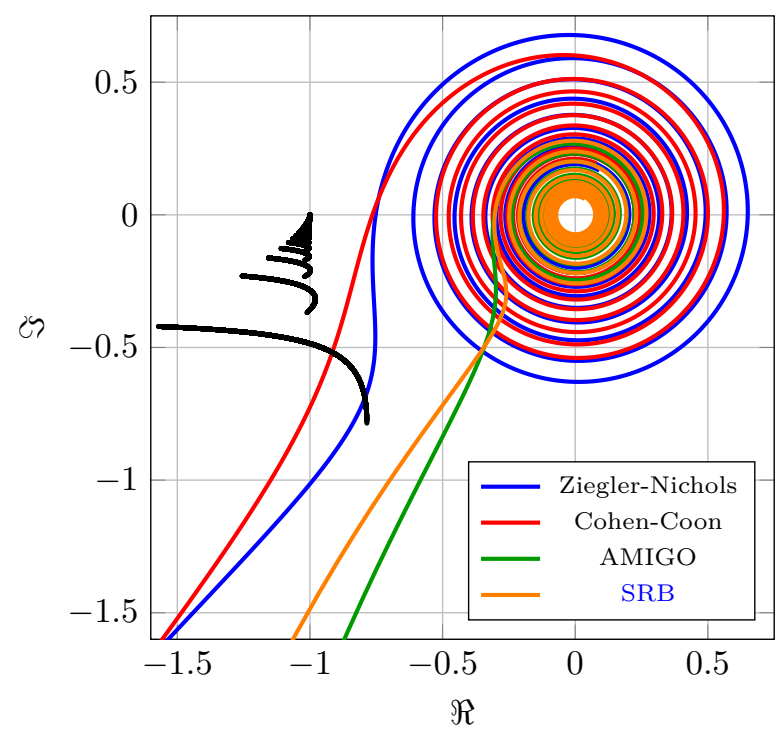

Figure 3: Plots of the open-loop transfer functions obtained with the PID parameters in Table 1 (colored lines) and $-1 / \mathcal{N}$ (black lines) in the Nyquist diagram.

\section{New robustness margin for existence of limit cycles}

\subsection{Tsypkin's method approach}

In order to predict more accurately the existence of limit cycles in the control systems presented in Figure 1 (or equivalently in Figure 2) and avoid them, an approach based on the Tsypkin's method [20] is proposed, which is valid independently of the filtering characteristics of the linear part of the system. Therefore, this approach is especially interesting for low order systems, as for example FOPTD systems that are considered in this paper.

In systems with a SSOD_ZOH nonlinearity as that shown in Figure 2, the general shape of an oscillation is depicted in Figure 5, in which the half period of an oscillation of $m$ levels is shown. In this figure, the error signal $e$ has been represented, whose slope may be of any magnitude. The sampled error signal $\bar{e}$ has also been plotted as a stair like signal with step widths defined by $\left(\rho_{p}-\rho_{p-1}\right) T_{o} / 2$, being $T_{o}$ the oscillation period and $\left.\rho_{i} \in\right] 0,1[$.

The Tsypkin's method formalizes the situation represented in Figure 5 in the set of mathematic relations that define the oscillation conditions presented in equations (3) and (4). 

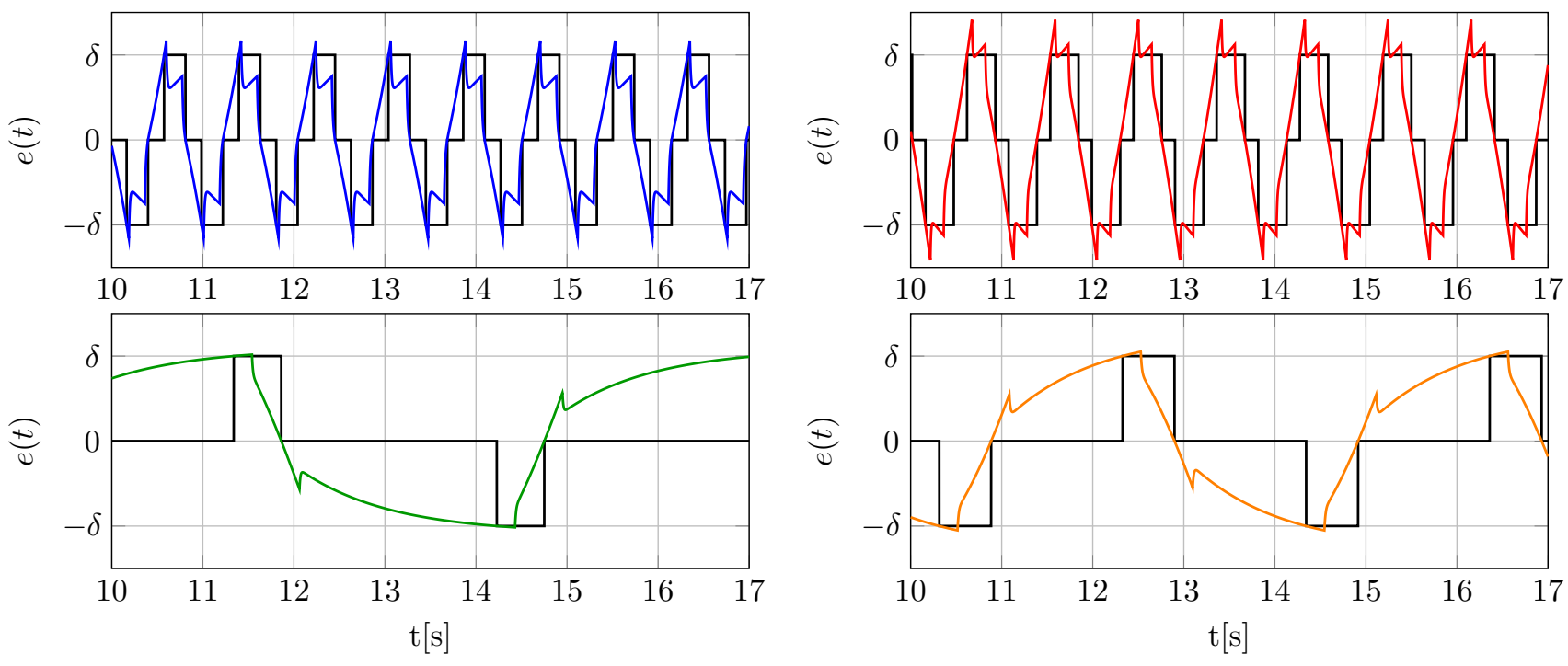

Ziegler-Nichols $\longrightarrow$ Cohen-Coon - AMIGO $\longleftarrow$ SRB

Figure 4: Steady state oscillations of the system with $G(s)$ given by equation (2) with the PID parameters in Table 1.

$$
\begin{gathered}
E C_{p} \mid e\left(\rho_{p} \frac{T_{o}}{2}\right)= \begin{cases}p \delta & \text { for } p=1,2 \ldots m \\
(2 m-p) \delta & \text { for } p=m+1, \ldots 2 m\end{cases} \\
I C_{p} \mid \frac{d e\left(\rho_{p} \frac{T_{o}}{2}\right)}{d t}= \begin{cases}>0 & \text { for } p=1,2 \ldots m \\
<0 & \text { for } p=m+1, \ldots 2 m\end{cases}
\end{gathered}
$$

These conditions are referred to the values of $e$ and its derivative in specific times $\rho_{p} \frac{T_{o}}{2}$, that is, when the thresholds of magnitude $p \delta$ are crossed. By applying the Fourier series and some basic calculations (see Appendix A) a general expression of $e\left(\rho_{p} \frac{T_{o}}{2}\right)$ can be obtained, equation (5), where $n$ is the number of harmonics:

$$
\begin{array}{r}
e\left(\rho_{p} \frac{T_{o}}{2}\right)=-\frac{4 \delta}{\pi} \sum_{n=1,3,5, \ldots}^{\infty} \frac{1}{n} \Re\left\{G_{o l}\left(j n \omega_{o}\right)\right\}\left(\sum_{i=1}^{m} i \sin \left(\frac{n \pi}{2}\left(\rho_{i+1}-\rho_{i}\right)\right) \cos \left(n \pi\left(\frac{\rho_{i+1}+\rho_{i}}{2}-\rho_{p}\right)\right)\right. \\
\left.+\sum_{i=m+1}^{2 m-1}(2 m-i) \sin \left(\frac{n \pi}{2}\left(\rho_{i+1}-\rho_{i}\right)\right) \cos \left(n \pi\left(\frac{\rho_{i+1}+\rho_{i}}{2}-\rho_{p}\right)\right)\right) \\
-\frac{4 \delta}{\pi} \sum_{n=1,3,5, \ldots}^{\infty} \frac{1}{n} \Im\left\{G_{o l}\left(j n \omega_{o}\right)\right\}\left(\sum_{i=1}^{m} i \sin \left(\frac{n \pi}{2}\left(\rho_{i+1}-\rho_{i}\right)\right) \sin \left(n \pi\left(\frac{\rho_{i+1}+\rho_{i}}{2}-\rho_{p}\right)\right)\right. \\
\left.+\sum_{i=m+1}^{2 m-1}(2 m-i) \sin \left(\frac{n \pi}{2}\left(\rho_{i+1}-\rho_{i}\right)\right) \sin \left(n \pi\left(\frac{\rho_{i+1}+\rho_{i}}{2}-\rho_{p}\right)\right)\right)
\end{array}
$$

Note that the previous expression depends on the real and imaginary part of the open-loop transfer function $\left(\Re\left\{G_{o l}\left(n \omega_{o}\right)\right\}, \Im\left\{G_{o l}\left(n \omega_{o}\right)\right\}\right)$ and on the number of levels $(m)$ crossed. It can be easily seen that as $m$ increases, the complexity of the calculations raise significantly because more oscillation conditions appear in equations (3) and (4), and the complexity of (5) increases too. For the purpose of this paper we will focus on single leveled oscillations $(m=1)$, because it has been observed that by avoiding oscillation for $m=1$ no oscillations for $m>1$ take place. This result will be proved in section 6 , where multi-level oscillations are addressed. Thus, for practical reasons, this study will be focused on oscillations with $m=1$, whose existence condition, obtained from equations (3) and (4) are as follows: 


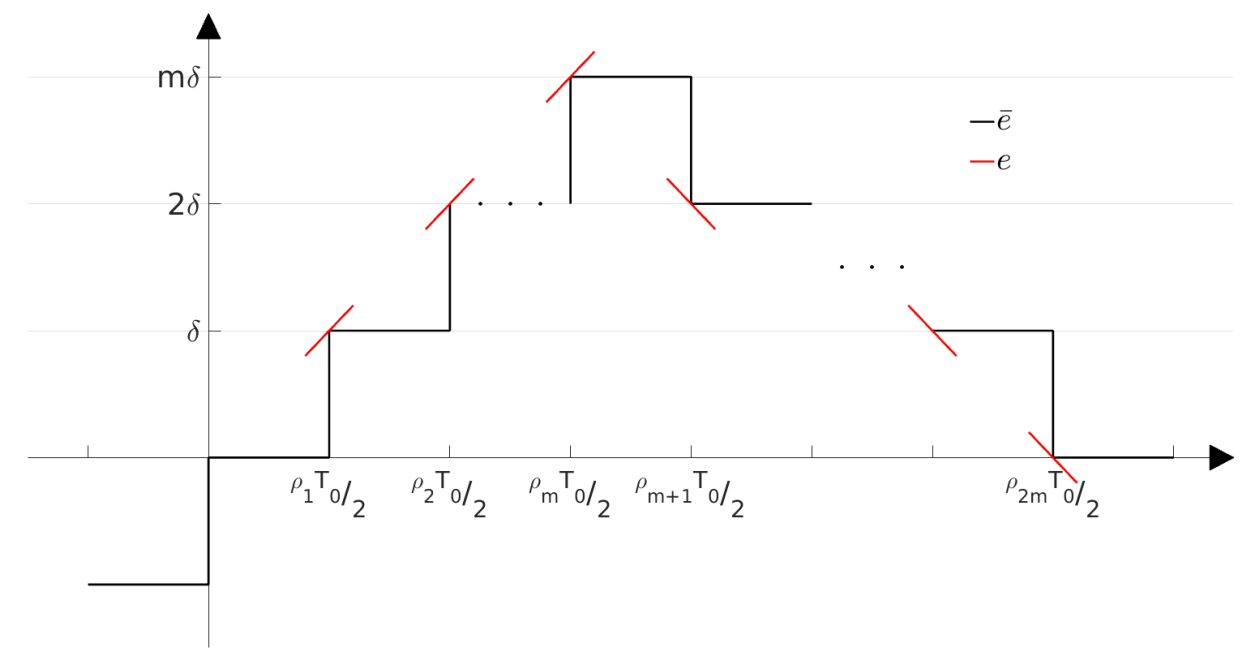

Figure 5: General form of an oscillation, which define the oscillation conditions in Tsypkin method.

$$
\begin{gathered}
E C_{p \mid m=1} \mid e\left(\rho_{p} \frac{T_{o}}{2}\right)= \begin{cases}\delta & \text { for } p=1 \\
0 & \text { for } p=2\end{cases} \\
I C_{p \mid m=1} \mid \frac{d e\left(\rho_{p} \frac{T_{o}}{2}\right)}{d t}= \begin{cases}>0 & \text { for } p=1 \\
<0 & \text { for } p=2\end{cases}
\end{gathered}
$$

It is worth noticing that conditions (6) and (7) are sufficient and necessary for the existence of limit cycles with $m=1$. Consequently, if any of the previous equations is not fulfilled, then, steady state oscillations do not appear in the system. Therefore, for the sake of simplicity and taking into account that the objective is to avoid limit cycles, we will consider the conditions in (6) to define a new robustness index to prevent oscillations. Evaluating (5) in $p=1,2$ and substituting the result in (6) taking into account that by definition $\rho_{2 m}=1$ and renaming $\rho_{1}$ as $\rho$, the following equations are obtained:

$$
\begin{array}{ll}
\sum_{n=1,3,5, \ldots}^{\infty} & \frac{1}{n} \Re\left\{G_{o l}\left(j n \omega_{o}\right)\right\} \sin (n \pi \rho)+\frac{\pi}{4}=0 \\
\sum_{n=1,3,5, \ldots}^{\infty} \frac{1}{n} \Im\left\{G_{o l}\left(j n \omega_{o}\right)\right\} \cos ^{2}\left(\frac{n \pi \rho}{2}\right)+\frac{\pi}{8}=0
\end{array}
$$

that finally can be rewritten as follows:

$$
\begin{gathered}
\Re\left\{G_{o l}\left(j \omega_{o}\right)\right\}=-\frac{\frac{\pi}{4}+\sum_{n=3,5 . .}^{\infty} \frac{1}{n} \sin (n \pi \rho) \Re\left\{G_{o l}\left(j n \omega_{o}\right)\right\}}{\sin (\pi \rho)} \\
\Im\left\{G_{o l}\left(j \omega_{o}\right)\right\}=-\frac{\frac{\pi}{8}+\sum_{n=3,5 . .}^{\infty} \frac{1}{n} \cos ^{2}\left(\frac{n \pi \rho}{2}\right) \Im\left\{G_{o l}\left(j n \omega_{o}\right)\right\}}{\cos ^{2}\left(\frac{\pi \rho}{2}\right)}
\end{gathered}
$$

For a given frequency $\omega_{o}$ and $\left.\rho \in\right] 0,1[$, the right hand members of the previous equations define a trajectory in the Nyquist plane what we refer to as Tsypkin branch for $\omega_{o}\left(B_{T}\left(\omega_{o}\right)\right)$.

$$
\left.B_{T}\left(\omega_{o}\right)=\left(-\frac{\frac{\pi}{4}+\sum_{n=3,5 . .}^{\infty} \frac{1}{n} \sin (n \pi \rho) \Re\left\{G_{o l}\left(j n \omega_{o}\right)\right\}}{\sin (\pi \rho)},-\frac{\frac{\pi}{8}+\sum_{n=3,5 . .}^{\infty} \frac{1}{n} \cos ^{2}\left(\frac{n \pi \rho}{2}\right) \Im\left\{G_{o l}\left(j n \omega_{o}\right)\right\}}{\cos ^{2}\left(\frac{\pi \rho}{2}\right)}\right), \quad \forall \rho \in\right] 0,1[
$$

Then, a limit cycle oscillation with frequency $\omega_{o}$ will take place if the Tsypkin branch for $\omega_{o}$ intercepts the point $\left(\Re\left\{G_{o l}\left(j \omega_{o}\right)\right\}, \Im\left\{G_{o l}\left(j \omega_{o}\right)\right\}\right)$ of the Nyquist diagram, that is: 


$$
\left(\Re\left\{G_{o l}\left(j \omega_{o}\right)\right\}, \Im\left\{G_{o l}\left(j \omega_{o}\right)\right\}\right)=B_{T}\left(\omega_{o}\right)
$$

Using these definitions we can establish a robustness measure against limit cycles as the minimum euclidean distance between the Nyquist points $\left(\Re\left\{G_{o l}(\omega)\right\}, \Im\left\{G_{o l}(\omega)\right\}\right)$ and its respective Tsypkin branches obtained for the same frequency $\omega$. We will refer to this robustness measure as Tsypkin margin $\left(M_{T}\right)$ that can be expressed as:

$M_{T}\left(\omega_{\min }, \rho_{\min }\right)=\sqrt{\left(\frac{\frac{\pi}{4}+\sum_{n_{\text {odd }}}^{\infty} \frac{1}{n} \sin \left(n \pi \rho_{\min }\right) \Re\left\{G_{o l}\left(j n \omega_{\min }\right)\right\}}{\sin \left(\pi \rho_{\text {min }}\right)}\right)^{2}+\left(\frac{\frac{\pi}{8}+\sum_{n_{\text {odd }}}^{\infty} \frac{1}{n} \cos ^{2}\left(\frac{n \pi \rho_{\min }}{2}\right) \Im\left\{G_{o l}\left(j n \omega_{\min }\right)\right\}}{\cos ^{2}\left(\frac{\pi \rho_{\min }}{2}\right)}\right)^{2}}$

being $\omega_{\min }$ and $\rho_{\min }$ the values of $\omega$ and $\rho$ for which the minimum distance is obtained.

The following examples illustrate the use of $M_{T}$ on predicting the existence of limit cycle and the graphical interpretation of this new robustness margin.

Example 2. Non-oscillatory system. Consider the following FOPTD transfer function:

$$
G(s)=\frac{e^{-s}}{s+1}
$$

A PI controller with $K_{p}=0.84$ and $T_{i}=1.17$ is tuned using the SRB method [19]. A set of Tsypkin branches represented in Figure 6 has been obtained by evaluating equations (9) and (10) for $\rho \in] 0,1\left[\right.$ and $\omega \in\left[\frac{\omega_{c g}}{200}, \omega_{c g}\right]$, where $\omega_{c g}$ is the crossover frequency. In order to make the visualization easier, different colors are used to represent each point $\left(\Re\left\{G_{o l}(j \omega)\right\}, \Im\left\{G_{o l}(j \omega)\right\}\right)$ and their respective $B_{T}(\omega)$. The minimal distance between $\left(\Re\left\{G_{o l}(j \omega)\right\}, \Im\left\{G_{o l}(j \omega)\right\}\right)$ and $B_{T}(\omega)$, which has been highlighted with a dashed line, is $M_{T}=0.23$, obtained for $\omega=1.0191 \mathrm{rad} / \mathrm{s}$. Note that even though there are several branches (the red ones) very close to some points of $G_{\text {ol }}$, these branches correspond to frequencies whose points $\left(\Re\left\{G_{o l}(j \omega)\right\}, \Im\left\{G_{o l}(j \omega)\right\}\right)$ (the red ones), are further than 0.23 .

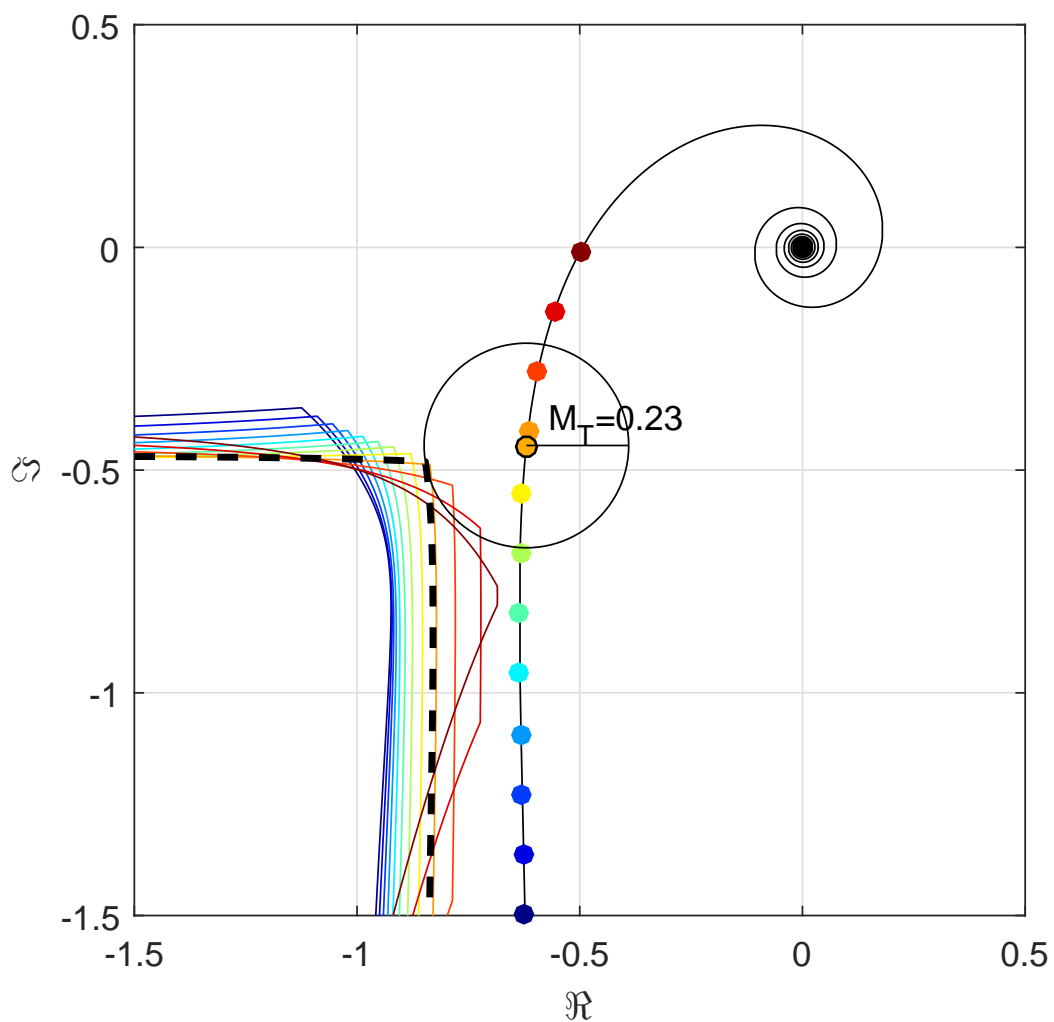

Figure 6: Nyquist diagram of $G_{o l}(\omega)$ and the Tsypkin band for a non oscillating system $\left(M_{T}=0.23\right)$. 


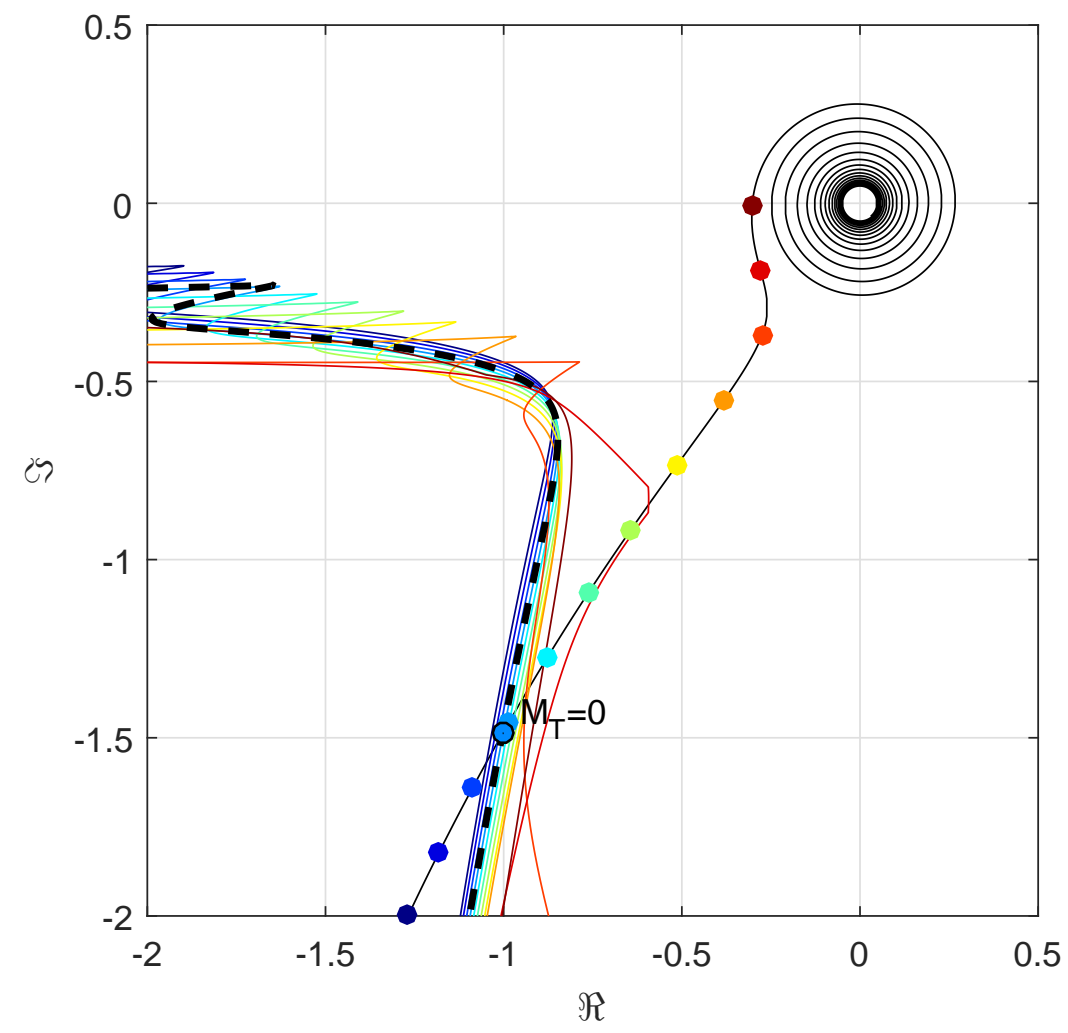

Figure 7: Nyquist diagram of $G_{o l}(\omega)$ and the Tsypkin band for a system with steady-state oscillations $\left(M_{T}=0\right)$.

Example 3. Oscillatory system. Consider the system in Example 1 and the PID obtained with SRB tuning procedure. Applying the concept of Tsypkin margin concludes that the system will oscillate because a distance $M_{T}=0$ has been obtained, as shown in Figure 7 . The intersection between $G_{o l}(j w)$ and $B_{T}(w)$ takes place for $\rho=0.72$ and $\omega_{o}=1.55 \mathrm{rad} / \mathrm{s}, B_{T}(1.55)$ has been represented with a dashed line. The simulation shown in Figure 4 confirms this prediction.

Example 4. $\mathbf{M}_{\mathbf{T}}$ for well known tuning rules. The robustness measure $M_{T}$ can be used to determine the robustness against the oscillations produced by the SSOD structure presented in Figure 1, regardless of the method used for tuning the controller $C(s)$. As an extension of the work presented in [12], $M_{T}$ has been evaluated for three well-known tuning methods, namely Ziegler-Nichols (ZN), Cohen-Coon (CC) and AMIGO tuning rules for both PI and PID. These methods have been used to tune controllers for FOPTD models with different ratio delay $(L)$ and time constant $(\tau)$. The results are summarized in Figure 8.

It can be seen that the AMIGO tuning rules offer higher values of $M_{T}$ with lower ratios $L / \tau$ and then this measure tends to a value around 0.45 , both for its PI and PID controller. The Ziegler-Nichols method offers lower values of $M_{T}$ than the AMIGO method for lower values of $L / \tau$, not being this the case for systems with higher ratio $L / \tau$ where the Ziegler-Nichols method offers the highest values of $M_{T}$. The Cohen-Coon method offers the lowest values of $M_{T}$ overall. It can be also observed that for all methods and controller types there are values of ratio $L / \tau$ with $M_{T}=0$. The range of values with $M_{T}=0$ is higher for PID controllers than for PI controller. Additionally, for each method the values of $M_{T}$ are higher for PI than for PID. This means that, in general, PI controllers have better robustness properties than PID when used with SSOD sampling strategy.

\section{SSOD-PID Controller Tuning}

In this section, we propose a new tuning method for SSOD-PID controllers. It is worth noting that most of the studies about SSOD based control systems are focused on the PI case and there is a lack of results concerning the event based PID algorithm. From the discussion on Example 4, it is clear that the PI controllers have better 


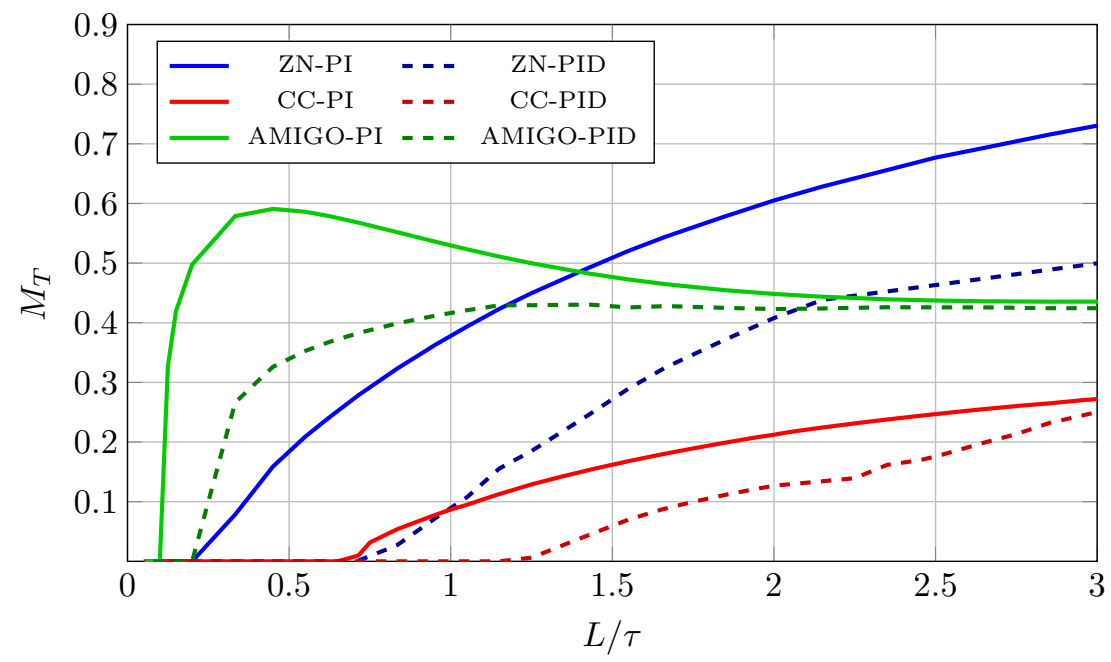

Figure 8: $M_{T}$ for FOPTD tuned with Ziegler-Nichols (ZN), Cohen-Coon (CC) and AMIGO tunning methods.

robustness properties than PID to be used on the control scheme presented in Figure 1. However, the benefits of PID algorithm on improving the response of the control systems is no matter of doubt, and the case of SSOD-PID is not an exception, as it will be proved in this section.

Our approach is focused on First Order Plus Time Delay (FOPTD) models, which allows to approximate a wide range of actual industry processes, including those systems that do not admit the application of tuning methods based on the describing function, as the ones presented in $[16,14]$, due to its low filtering properties. When using FOPTD models a good technique for generalizing the results is expressing the transfer function in dimensionless form. Our proposal is based on this feature, so before presenting the tuning method some preliminary results about the dimensionless approach are presented in the next subsection.

\subsection{Preliminary issues on dimensionless analysis}

Let us consider $G(s)$ in Figure 2 to be a FOPTD model. Without loss of generality, the network delay $t_{d}$ can be included in the time delay term $L$.

$$
G(s)=\frac{K e^{-L s}}{\tau s+1}
$$

A dimensionless representation of $G(s)$ is attained by making the transformation $\bar{s}=L s$ and $G(\bar{s}) / K$ :

$$
G(s)=\frac{K e^{-L s}}{\tau s+1} \stackrel{s=\frac{\bar{s}}{L}}{\Longrightarrow} G(\bar{s})=\frac{K e^{-\bar{s}}}{\frac{\tau}{L} \bar{s}+1} \stackrel{G(\bar{s}) / K}{\Longrightarrow} \bar{G}(\bar{s})=\frac{e^{-\bar{s}}}{\frac{\tau}{L} \bar{s}+1}
$$

The use of the dimensionless form of $G(s)$ makes the analysis easier because the results can be expressed in terms of the ratio $L / \tau$, the only parameter of $\bar{G}(\bar{s})$. That is the case for homogeneous tuning rules for PID controllers which can be expressed by the following equations:

$$
K_{p}=K^{-1} \phi_{1}\left(\frac{L}{\tau}\right)=K^{-1} \bar{K}_{p}, \quad T_{i}=L \phi_{2}\left(\frac{L}{\tau}\right)=L \bar{T}_{i}, \quad T_{d}=L \phi_{3}\left(\frac{L}{\tau}\right)=L \bar{T}_{d}
$$

Taking into account the previous equations, the dimensionless transfer function of the PID, obtained from equation (1), is:

$$
C(s)=K_{p}\left(1+\frac{1}{T_{i} s}+\frac{T_{d} s}{\frac{T_{d} s}{N}+1}\right) \stackrel{s=\frac{\bar{s}}{L}}{\Longrightarrow} C(\bar{s})=K_{p}\left(1+\frac{1}{T_{i} / L \bar{s}}+\frac{T_{d} / L \bar{s}}{\frac{T_{d} / L \bar{s}}{N}+1}\right)
$$




$$
\bar{C}(\bar{s})=K C(\bar{s}) ; \quad \bar{C}(\bar{s})=\phi_{1}\left(1+\frac{1}{\phi_{2} \bar{s}}+\frac{\phi_{3} \bar{s}}{\frac{\phi_{3} \bar{s}}{N}+1}\right)
$$

where, for the sake of simplicity, the argument $L / \tau$ has been suppressed from $\phi_{1}, \phi_{2}$ and $\phi_{3}$. The dimensionless open-loop transfer function of the system in Figure 2 can be obtained using equations (12) and (15):

$$
\overline{G_{o l}}(\bar{s})=\bar{C}(\bar{s}) \bar{G}(\bar{s})=\phi_{1}\left(1+\frac{1}{\phi_{2} \bar{s}}+\frac{\phi_{3} \bar{s}}{\frac{\phi_{3} \bar{s}}{N}+1}\right) \frac{e^{-\bar{s}}}{\frac{\tau}{L} \bar{s}+1}
$$

Furthermore, from equations (12) and (15):

$$
\overline{G_{o l}}(\bar{s})=\bar{C}(\bar{s}) \bar{G}(\bar{s})=(K C(\bar{s}))\left(\frac{G(\bar{s})}{K}\right)=C(\bar{s}) G(\bar{s})=G_{o l}(\bar{s})=G_{o l}(s L)
$$

The use of homogeneous rules for tuning the PID controllers has important implications that can simplify the design of SSOD-PID for FOPTD systems. Concerning $M_{T}$, since its value only depends on the open-loop transfer function, and according to the equation $(17) \overline{G_{o l}}(\bar{s})=G_{o l}(s L)$, the value of Tsypkin margin calculated with $\overline{G_{o l}}(\bar{s})$, denoted as $\overline{M_{T}}$, holds the following relation with $M_{T}$ :

$$
\overline{M_{T}}\left(\overline{\omega_{\min }}, \rho_{\min }\right)=M_{T}\left(\omega_{\min } L, \rho_{\min }\right)
$$

Thus, for FOPTD models tuned with homogeneous tuning rules, evaluating $M_{T}$ with the dimensionless openloop transfer function $\overline{G_{o l}}(\bar{s})$ offers the same value of $M_{T}$ than with the dimensional open-loop transfer function. Moreover, the frequency $\omega_{\min }$ can be directly obtained from the dimensionless value $\overline{\omega_{\min }}$ as $\overline{\omega_{\min }}=\omega_{\min } L$.

When using a PID controller in structures with a SSOD sampler, other important issue to be considered in the design is the change $\delta_{u}$ in the control action produced by the changes $\delta$ on the sampled error signal $\bar{e}$. The value of $\delta_{u}$ is directly related to the smoothness of the control action and its limitation is important to avoid sudden changes in the control action that could harm the actuator or cause a malfunctioning of the control system. It should be taken into account that $\bar{e}$ is a noise free stair like signal with steps of magnitude $\delta$, which are amplified by the controller in the same magnitude as a high frequency noise, according the following equation:

$$
\delta_{u}=K_{p}(1+N) \delta=C(\infty) \delta,
$$

where $K_{p}$ is the controller gain and $N$ the derivative filter coefficient. Concerning the selection of $N$ it is important to note that although a common practice in industry is to fix it at high values, some researches have recently shown that using $N$ as a free parameter allows to balance the noise amplification and the closed loop performance (see $[10,11])$. A similar effect of $N$ in the case of the event based system in Figure 1 has been reported in [17], where the selection of this parameter allows to set a trade-off between the control action jumps $\delta_{u}$ and the IAE of the disturbance response.

The fact of using homogeneous tuning rules, allows to obtain the value of $C(\infty)$ for a given system from the dimensionless model by dividing the obtained value of $\bar{C}(\infty)$ by the system gain:

$$
C(\infty)=K_{p}(1+N)=\frac{\phi_{1}}{K}(1+N)=\frac{\bar{C}(\infty)}{K}
$$

Finally, following the dimensionless approach, a simple relation can be extracted between the $I A E$ index for dimensional and dimensionless models, which is given by the following equation (see Appendix B):

$$
I A E=|K| L \cdot \overline{I A E}
$$

In summary, when using homogeneous rules to tune a PID controller for a FOPTD system $G(s)$, the value of the controller parameters, $C(\infty)$ and $I A E$ (or other indexes) can be calculated by scaling the results obtained with the dimensionless model $\bar{G}(\bar{s})$ using the equations (13), (20) and (21) respectively. Furthermore, the value of $M_{T}$ is the same as that obtained with $\bar{G}(\bar{s})$. To illustrate this idea let us introduce the Example 5 . 
Table 2: Summary of characteristics for the studied systems.

\begin{tabular}{|c|c|c|c|c|c|c|c|c|}
\hline & \multicolumn{4}{|c|}{ PI } & \multicolumn{4}{|c|}{$\operatorname{PID}(\mathbf{N}=\mathbf{1 0})$} \\
\hline & $\mathbf{M}_{\mathbf{T}}$ & IAE $_{\mathbf{p}}$ & $\mathbf{I A E}_{\mathbf{r}}$ & $\mathbf{C}(\infty)$ & $\mathbf{M}_{\mathbf{T}}$ & $\mathbf{I A E}_{\mathrm{p}}$ & $\mathbf{I A E}_{\mathbf{r}}$ & $\mathbf{C}(\infty)$ \\
\hline$\overline{\mathbf{G}}(\overline{\mathbf{s}})$ & 0.38 & 3.3168 & 3.3458 & 0.9 & 0.0927 & 1.6626 & 1.9092 & 13.2 \\
\hline$G_{1,2}(s)$ & 0.38 & 6.6335 & 6.6916 & 0.9 & 0.0981 & 3.253 & 3.8184 & 13.2 \\
\hline$G_{2,1}(s)$ & 0.38 & 6.6335 & 3.3458 & 0.45 & 0.0927 & 3.253 & 1.9092 & 6.6 \\
\hline$G_{2,2}(s)$ & 0.38 & 13.2671 & 6.6916 & 0.45 & 0.0981 & 6.6505 & 3.8184 & 6.6 \\
\hline
\end{tabular}

Example 5. Let us consider three different FOPTD systems whose transfer functions are

$$
G_{1,2}(s)=\frac{e^{-2 s}}{2 s+1}, \quad G_{2,1}(s)=\frac{2 e^{-s}}{s+1}, \quad G_{2,2}(s)=\frac{2 e^{-2 s}}{2 s+1} .
$$

The dimensionless model for these systems is $\bar{G}(\bar{s})=\frac{e^{-\bar{s}}}{\bar{s}+1}$. PI and PID controllers are tuned using the ZieglerNichols method, which is a well-known homogeneous tuning rule. The resulting $M_{T}$, the disturbance IAE (IAE $E_{p}$ ), the reference IAE $\left(I A E_{r}\right)$ and $C(\infty)$ of each system is computed. The results are gathered in Table 2.

This table shows how all the systems, which have the same ratio $L / \tau$, have the same value of $M_{T}$, which confirms that this margin only depends on the ratio $L / \tau$. The relation of the other performance indexes (IAE $E_{p}, I A E_{r}$ and $C(\infty))$ with their dimensionless counterparts fulfill the described relations, as expected.

\subsection{Tunning procedure}

According to the previous section, it is possible to calculate the controller parameters for any FOPTD transfer function $G(s)$ with a given quotient $L / \tau$ and evaluate its performance and robustness from the results obtained for the dimensionless model $\bar{G}(\bar{s})$ with the same value of $L / \tau$. In this section, we provide the controller parameters and performance values for a wide range of models $\bar{G}(\bar{s})$ taking into account an optimum tradeoff between the $\overline{I A E}$ performance index, the smoothness of the control action in terms of $\bar{C}(\infty)$ and the robustness measure to limit cycle $M_{T}$. From these values, it is possible to calculate the results for any system $G(s)$ with the same ratio $L / \tau$ using the equations (13), (20) and (21).

Our approach is based on the tuning algorithm proposed in [19], that we call SRB method, which minimize the disturbance $I A E$ index with restrictions on the phase and gain margins. The selection of this algorithm is based on three facts: 1) This method offers different robustness configurations expressed in terms of phase and gain margins. The definition of $M_{T}$ intuitively suggests a direct relation with the phase margin. Therefore, different values of $M_{T}$ can be obtained by changing the phase margin. 2) The derivative filtering coefficient $N$ is considered as a free design parameter, offering controllers with a smoother control action than those with high values of $N$. 3) According to [3], the minimization of the disturbance IAE, which depends on the plant sensitivity, assures the homogeneity of the tuning results obtained with this method.

It is worth noticing that SRB method has been originally developed for continuous-time controllers, but our objective is to extend it to systems with a SSOD sampling strategy, the one presented in Figure 1, by fulfilling the requirements on $M_{T}$ and $C(\infty)$. More precisely, the design must minimize the disturbance $I A E$ while meeting requirements on robustness to limit cycle $\left(M_{T}>M_{T_{r}}\right)$ and smoothness of the control action $\left(C(\infty)<C_{r}(\infty)\right)$ that formally can be written as in equation (22). Nevertheless, the relation between these requirements and the design parameters used in the SRB method, that involves phase and gain margins $\left(\phi_{m}, \gamma_{m}\right)$ and the derivative filtering coefficient $(N)$, is not evident.

$$
\begin{array}{ll}
\underset{K_{p}, T_{i}, T_{d}, N}{\operatorname{minimize}} & I A E=\int_{0}^{\infty}|e(t)| d t \\
\text { subject to } & M_{T} \geq M_{T_{r}} \\
& C(\infty) \leq C_{r}(\infty)
\end{array}
$$

The proposed solution is to calculate the controllers using various combinations of phase and gain margins and derivative filtering coefficient. This produces a set of controllers with $M_{T}, \overline{I A E}, \bar{C}(\infty)$ and parameters $\overline{K_{p}}, \overline{T_{i}}$, 


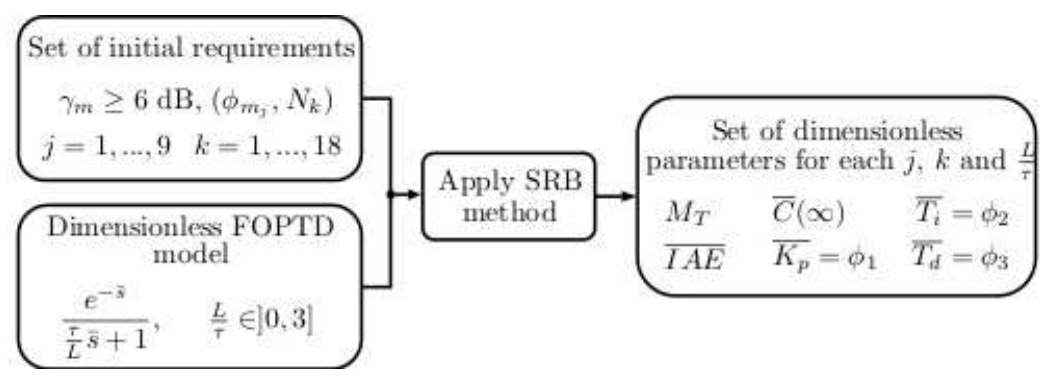

Figure 9: Summary of the calculus to obtain the dimensionless set of parameters for the controllers.

$\overline{T_{d}}$. Then, the controller that minimizes $I A E$ fulfilling $M_{T}>M_{T_{r}}$ and $C(\infty)<C_{r}(\infty)$ is easily selected from this set. The validity of this approach lies on the fact that once the controllers are obtained for a batch of $\bar{G}(\bar{s})$, that is, varying the parameter $L / \tau$, the results can be used to obtain the controller for any FOPTD model with $L / \tau$ contained in the batch.

\subsection{Calculating the dimensionless controllers}

Following the previously described approach, dimensionless parameters were calculated using the SRB method for a set of systems $\bar{G}(\bar{s})$ with $L / \tau \in] 0,3]$ for all the possible combinations of $\phi_{m}=\left[50^{\circ}, 52.5^{\circ}, 55^{\circ}, 57.5^{\circ}, 60^{\circ}, 62.5^{\circ}\right.$, $\left.65^{\circ}, 67.5^{\circ}, 70^{\circ}\right]$ and $N=[0,0.5,1,2,3,4,5,6,7,8,9,10,12,14,15,16,18,20]$. The gain margin was required to meet $\gamma_{m} \geq 6 \mathrm{~dB}$. The procedure is summarized in Figure 9 . The values of $\phi_{m}$ and $N$ have been selected to guarantee a set of controllers dense enough in order to make the final controller as close as possible to the exact controller that minimizes the IAE under the restrictions in $M_{T}$ and $C(\infty)$, that is, the solution of the optimization problem given by equation (22). As said in the previous section, once the set of dimensionless parameters is calculated, it can be used to obtain the controller for any FOPTD model with $L / \tau \in] 0,3]$, in consequence, the proposed griding of $\phi_{m}$ and $N$ does not suppose a computation overload for the tuning of each controller. It is also important to remark that the phase and gain margins used as requirements are maintained from the dimensionless model to the dimensional one, the only difference between the dimensionless and the dimensional cases is the frequency at which these margins are measured due to the variable change $\left(j \overline{\omega_{\min }}=j \omega_{\min } L\right)$. Therefore, in addition to the required characteristics $M_{T_{r}}$ and $C_{r}(\infty)$, all the controllers of the set have at least $\phi_{m}=50^{\circ}$ and $\gamma_{m}=6 \mathrm{~dB}$.

Figure 10 shows the evolution of the margin $M_{T}$ with the ratio $L / \tau$ for some representative values of $\phi_{m}$ and $N$. Several interesting conclusions rise from this figure. Firstly, it can be seen that for systems close to a first order model (low values of $L / \tau$ ),$M_{T}=0$. Then, for given values of $L / \tau$, depending on $\phi_{m}$ and $\gamma_{m}, M_{T}$ increases quickly, reaching its maximum and then decreasing asymptotically to a final value. Secondly, the figures show that the higher the phase margin, the higher $M_{T}$. This confirms our intuition about the relation between these parameters, based on the fact that both measures are related with the robustness of the system. Finally, the relation between the filtering coefficient $N$ and the robustness to limit cycle is clearly shown: increasing the value of $N$ generally reduces $M_{T}$. In addition, it can be seen that it does not exist much difference between the values of $M_{T}$ obtained for $N=10,15$ and 20, thus, regarding to $M_{T}$, increasing $N$ in a range further than 10 does not change significantly the robustness of the system. Summarizing, in the majority of the considered cases, incrementing $\phi_{m}$ tends to increase $M_{T}$ but increasing $N$ tends to decrease $M_{T}$.

Figures 11 and 12 show the values of $\overline{I A E}$ and $\bar{C}(\infty)$ for different values of $L / \tau$. Only the cases for which $M_{T} \neq 0$ have been represented. The worst results of $\overline{I A E}$ are obtained for the PI controllers $(N=0)$ and this index improves as $N$ rises, however, as in the case of $M_{T}$, no significant improvement is observed regarding to the $I A E$ for those controllers with values of $N>10$. On the other hand, the effect of $\phi_{m}$ on the $\overline{I A E}$ is not significant for the PID controllers, for any value of $N$. The same can not be said for the PI cases, whose values of $\overline{I A E}$ for low ratios $L / \tau$ clearly get worse with the increment of $\phi_{m}$. Regarding $\bar{C}(\infty)$, it can be seen how, as expected, increasing $N$ increases $\bar{C}(\infty)$, which increases significantly the value of $\delta_{u}$. Furthermore, the variations of $\phi_{m}$ barely affect this parameter.

\subsection{Software tool for tuning}

As a result of the calculation described on the previous section, 162 controllers $\left(\overline{K_{p}}, \overline{T_{i}}\right.$ and $\left.\overline{T_{d}}\right)$ have been obtained for each value of $L / \tau \in] 0,3]$, by taking all the possible combinations of $\phi_{m}=\left[50^{\circ}, 52.5^{\circ}, 55^{\circ}, 57.5^{\circ}, 60^{\circ}\right.$, 

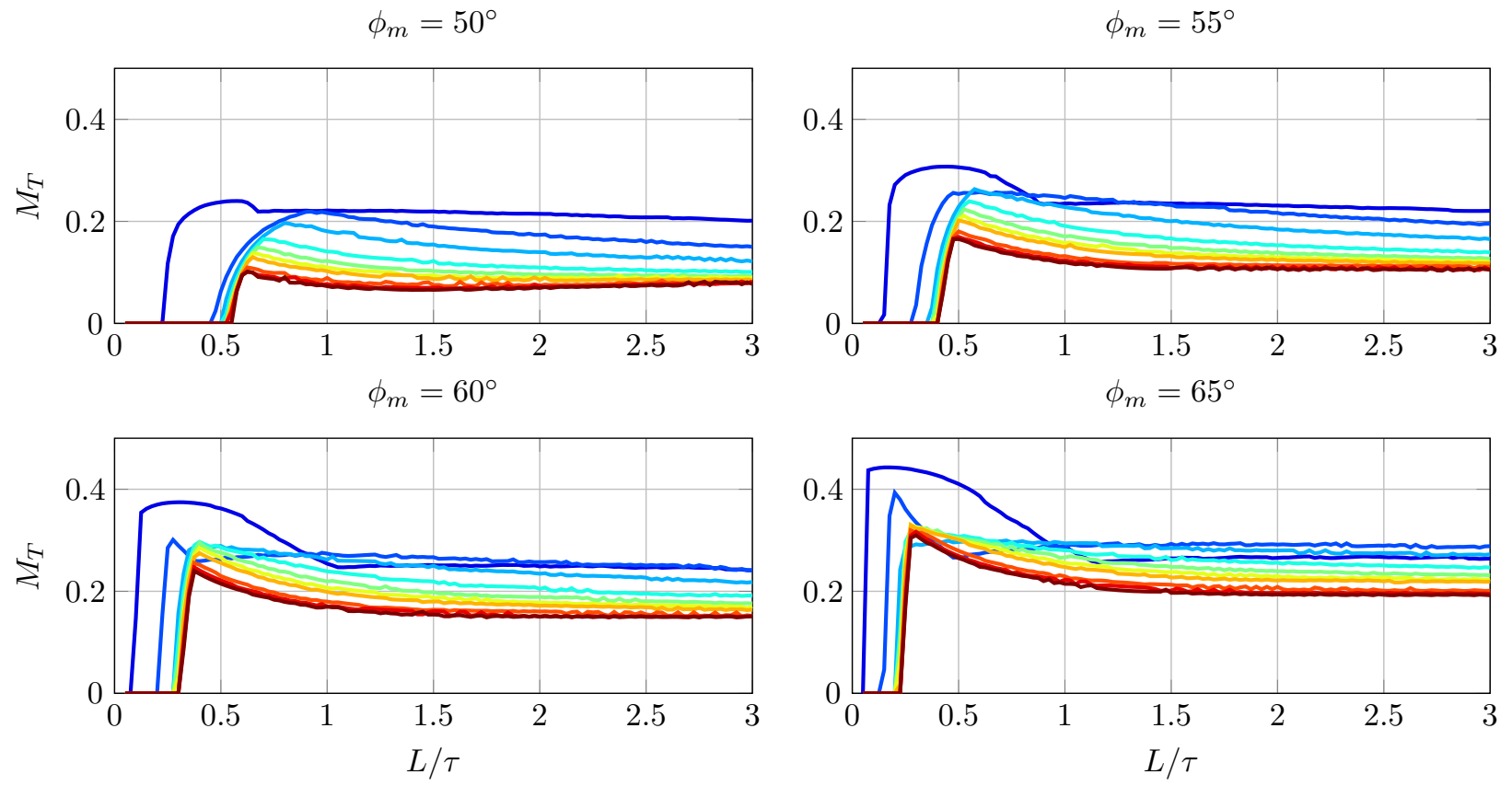

$\longrightarrow \mathrm{N}=0 \longrightarrow \mathrm{N}=0.5-\mathrm{N}=1-\mathrm{N}=2-\mathrm{N}=3-\mathrm{N}=4-\mathrm{N}=5 \quad \mathrm{~N}=10 \quad \mathrm{~N}=15 \longrightarrow$

Figure 10: Evolution of $M_{T}$ with $L / \tau$ for different configurations of $\phi_{m}$ and $N$.
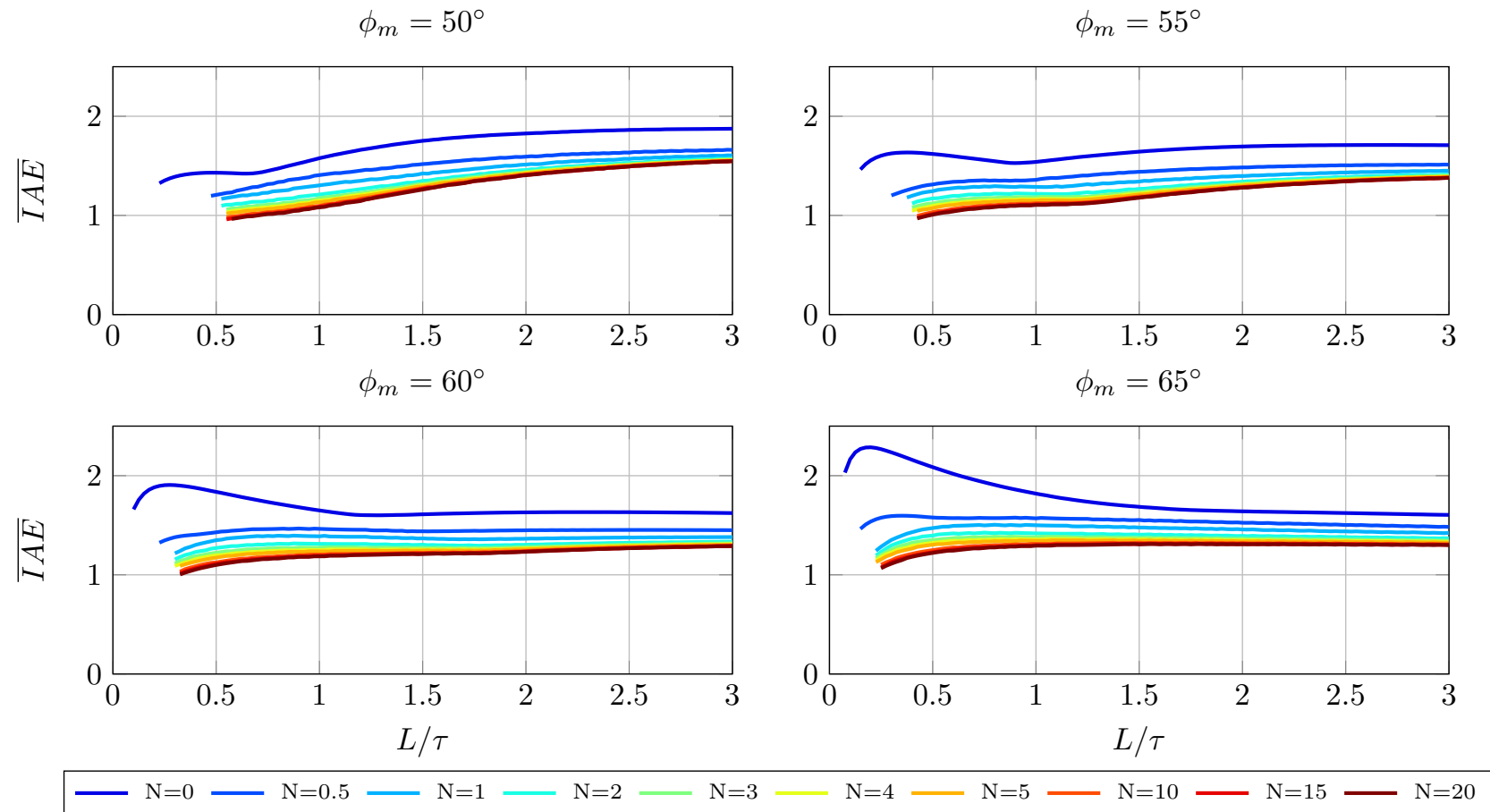

Figure 11: Obtained values of $\overline{I A E}$ for the considered batch for different configurations of $\phi_{m}$ and $N$. 

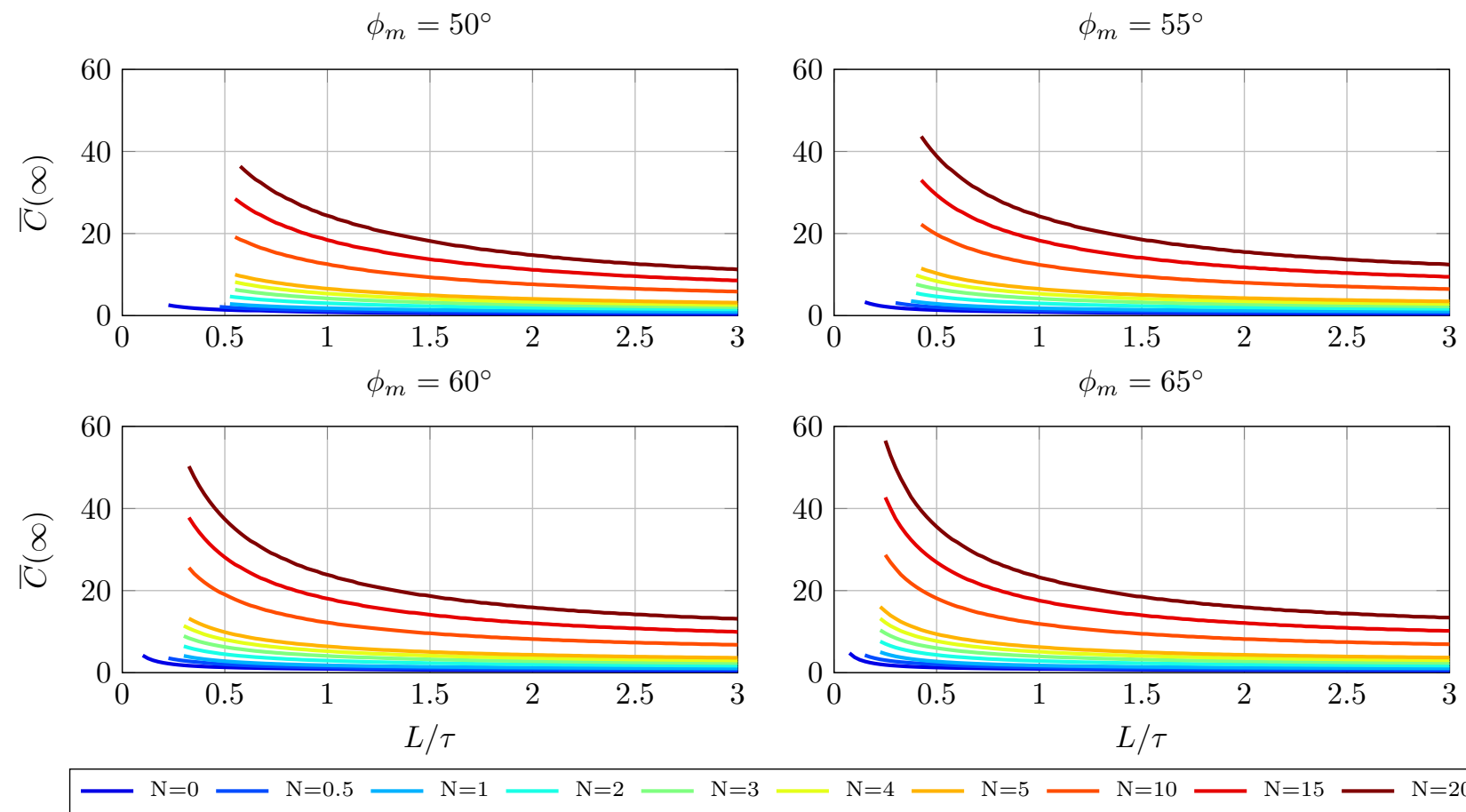

Figure 12: Obtained values of $\bar{C}(\infty)$ for the considered batch for different configurations of $\phi_{m}$ and $N$.

$\left.62.5^{\circ}, 65^{\circ}, 67.5^{\circ}, 70^{\circ}\right]$ and $N=[0,0.5,1,2,3,4,5,6,7,8,9,10,12,14,15,16,18,20]$. Their respective values of $M_{T}, \overline{I A E}$ and $\bar{C}(\infty)$ have also been obtained. In order to manage this information more efficiently, a software tool has been developed to facilitate the selection of the controller that minimizes the $I A E$ while fulfilling the constraints $M_{T}>M_{T_{r}}$ and $C(\infty)<C_{r}(\infty)$. It should be noted in Figures 10, 11 and 12 that smooth relations exist between the design parameters $\left(\phi_{m}\right.$ and $\left.N\right)$ and the robustness/performance indexes $\left(M_{T}, \overline{I A E}\right.$ and $\left.\bar{C}(\infty)\right)$. This indicates that the proposed tuning procedure is not too sensitive to these parameters and therefore the solution obtained from the pre-calculated set of dimensionless controllers will be close to the actual optimal solution.

The tuning procedure using the set of dimensionless parameters pre-calculated in section 4.3 has been implemented in a Java application. The procedure is summarized in Figure 13. For a given FOPTD model and requirements $M_{T_{r}}$ and $C_{r}(\infty)$ the quotient $L / \tau$ is calculated and the controllers for this value of $L / \tau$ fulfilling the constrains $M_{T}>M_{T_{r}}$ and $\bar{C}(\infty) / K<C_{r}(\infty)$ are filtered. Then the controller with minimum $\overline{I A E}$ is selected and the dimensional parameters $K_{p}, T_{i}$ and $T_{d}$ are calculated using equations (13). If an arbitrary model is defined, the tool first obtains a FOPTD approximation, and calculates the controller using that approximation. The robustness margins and the response simulation, however, correspond to the original system, therefore it is easy to check the effect of approximate modeling on the design. The software tool can be freely downloaded from:

https://sites.google.com/a/uji.es/freepidtools/ssodTsypkinPid.

The use of the application is introduced through the following example.

Example 6. Let us consider for this example a system whose transfer function is:

$$
G(s)=\frac{1}{\left(s+\frac{1}{3}\right)(s+1)(s+3)}
$$

The application consists of a main window including several tabs with different functionality. Firstly, the process model must be defined in the Plant_definition tab, Figure 14. As commented before, since this model is not a FOPTD transfer function, a FOPTD approximation is calculated which will be used to obtain the controller parameters. The PID design is carried out in the PID_design tab, Figure 15, by fixing the desired value $M_{T_{r}}$ and $C(\infty)$. For this example it has been considered $M_{T_{r}} \geq 0.1$ and $C(\infty) \leq 20$. Then a button allows to obtain the PID controller parameters that minimize the IAE while fulfilling these constraints. The design results evaluated over 


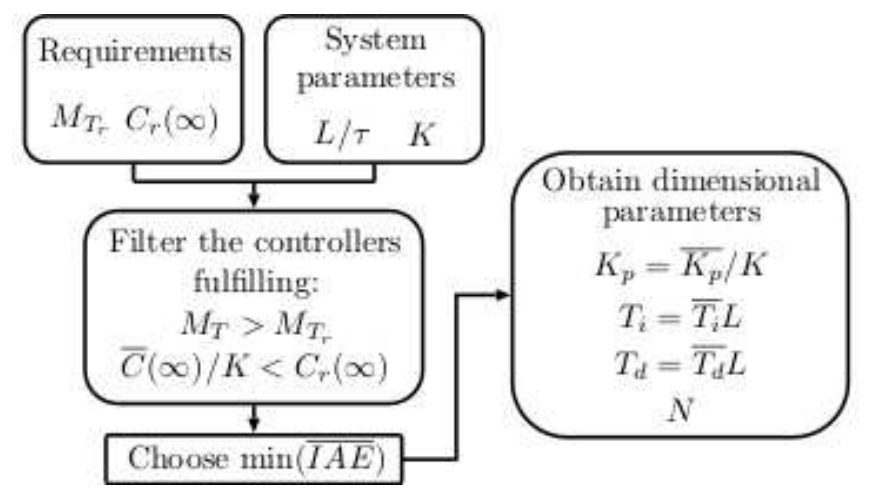

Figure 13: Summary of the controller design procedure.

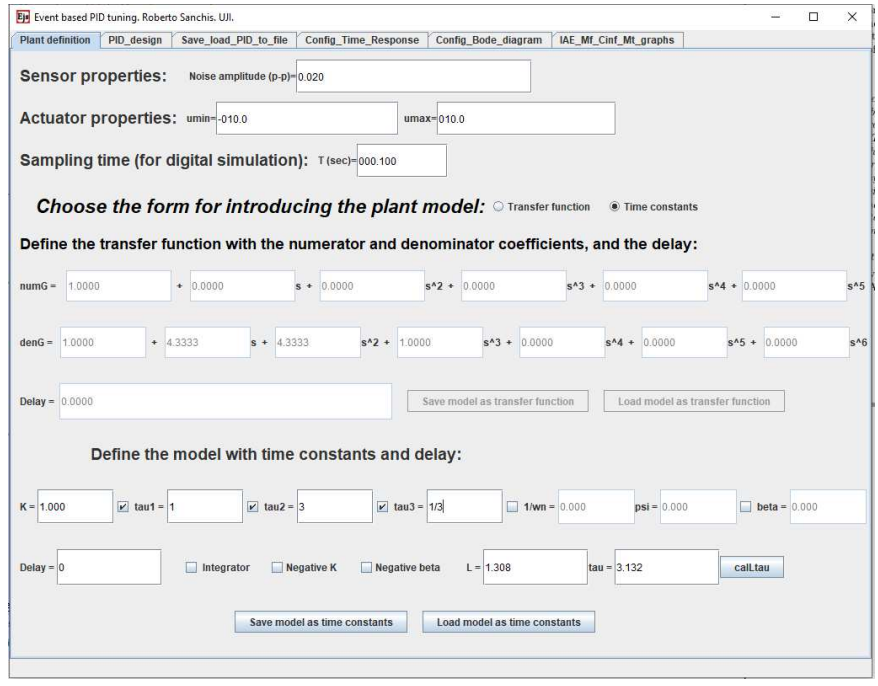

Figure 14: Software tool process definition window.

the original systems, not the FOPTD approximation, are also shown on this tab. It can be seen how $M_{T}=0.32$ is greater than $M_{T_{r}}$ and that the value of $C(\infty)=18.28$ stays below the maximum required. The tool also shows the Tsypkin band that defines the margin $M_{T}$, and the complete set of Tsypkin bands if desired.

A separate window shows the response of the controlled system to a step change in the setpoint and in the disturbance input, see Figure 16, allowing to select the value of the SSOD parameter $\delta$, which has been fixed to 0.1.

If the user wants to find another controller, the new restrictions must be introduced and the application will compute the result. For example, consider now that the requirement on the control action changes to $C_{r}(\infty) \leq 2$, then this parameter is introduced and the application computes the resultant controller as it is shown in Figure 17. Here it can be seen that the new controller, which is a PI controller, fulfills the requirements on $M_{T}$ and $C(\infty)$, but the disturbance IAE has risen (from 1.37 to 1.86), as can be appreciated on Figure 18.

\subsection{Tuning table}

Alternatively to the software tool presented in the previous section, the tuning can also be carried out using the table in Appendix $\mathrm{C}$, that has been obtained for specific requirements on $\bar{C}(\infty)$ and $M_{T_{r}}>0.2$. Of course, this option is not as flexible and interactive as using the application, but provides a very simple way for tuning the controller once the FOPTD model of the system is known.

The table provides the dimensionless parameters $\overline{K_{p}}, \overline{T_{i}}$ and $N$ for different values of the ratio $L / \tau$. The value of $\overline{T_{d}}$ has been omitted since its value is calculated as $\overline{T_{d}}=\overline{T_{i}} / 4$. The empty entries in the table mean that it is not possible to obtain a controller with the SRB method that fulfills the specified requirements. For the entries where a character $*$ appears, the controller parameters are the same as those obtained for the precedent value of $\bar{C}(\infty)$, 


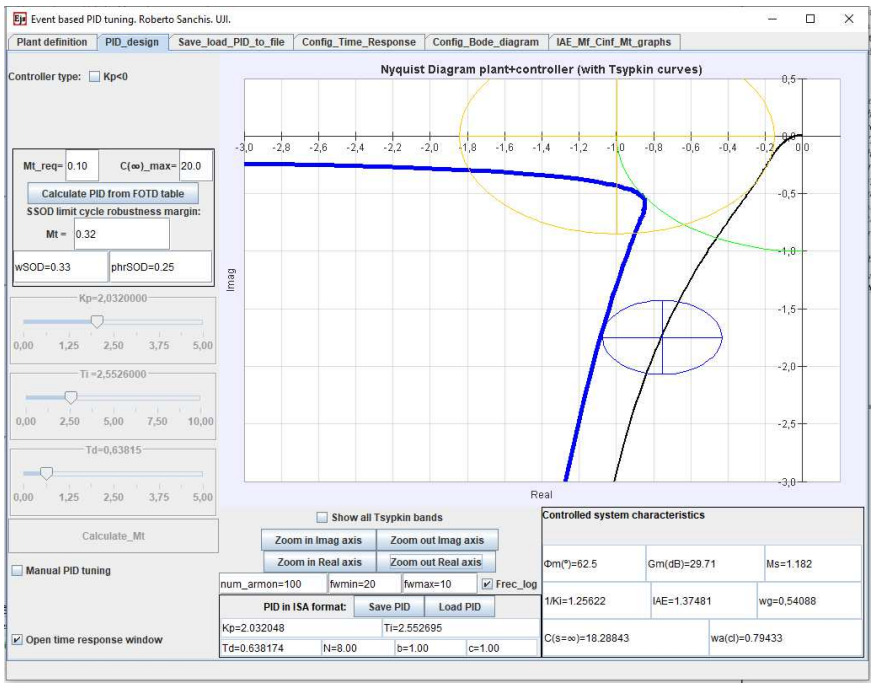

Figure 15: Software tool PID design window.

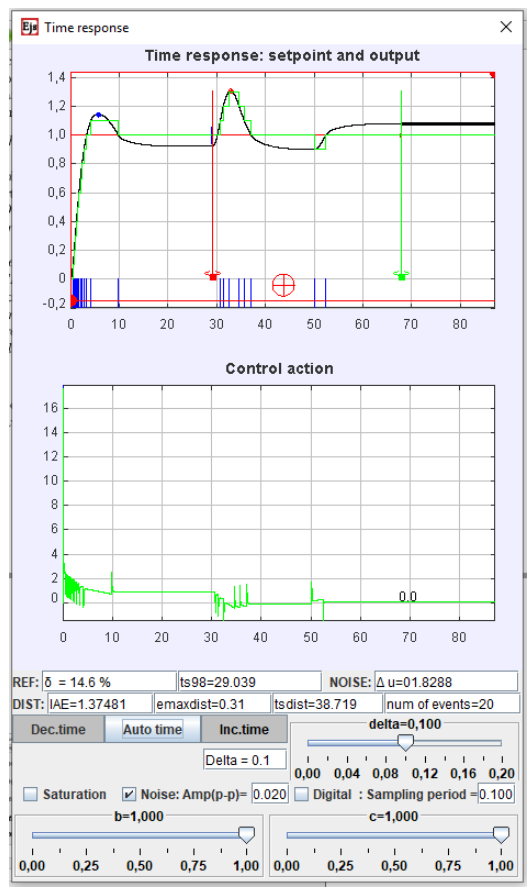

Figure 16: Software tool time response window. 


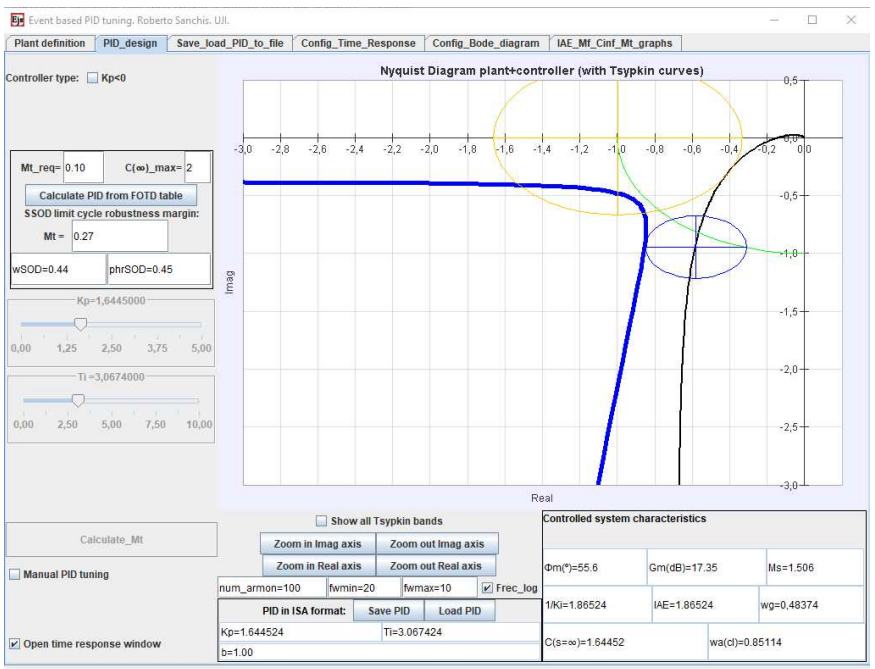

Figure 17: Software tool PID design window with modified parameters.

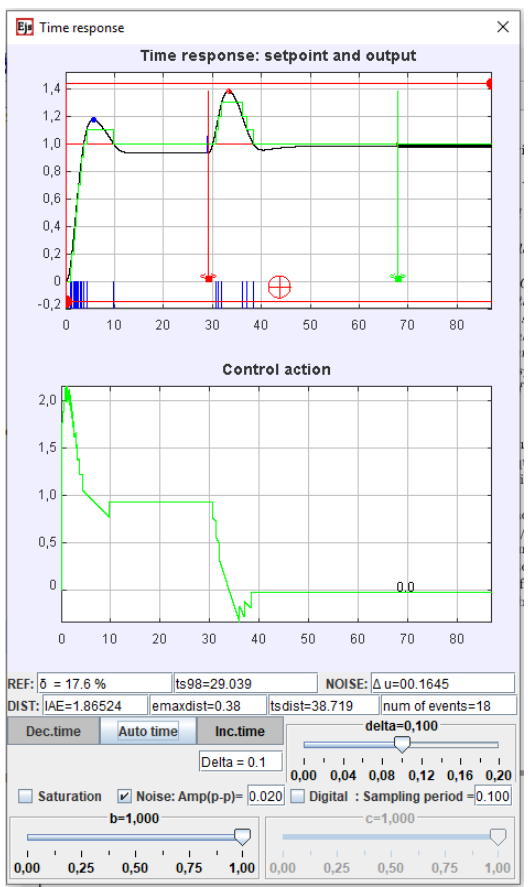

Figure 18: Software tool time response window with modified parameters. 

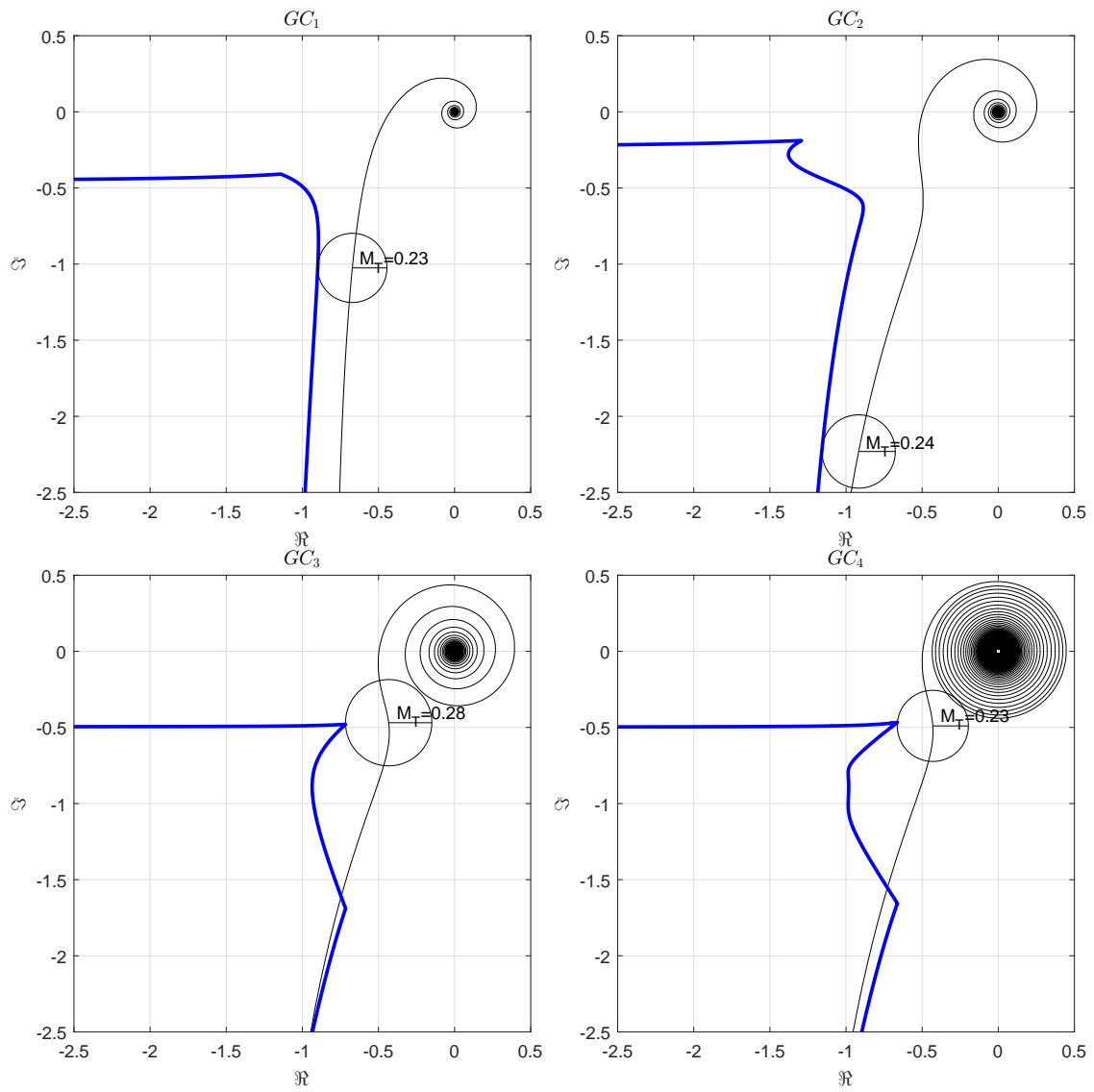

Figure 19: Nyquist plot of the open-loop transfer function with all the controllers and its minimum Tsypkin branch.

that is, the parameters on the columns at the left of the position marked with $*$. The following example illustrates how the different proposed controllers on the table behave and some guidelines to choose them.

Example 7. Let us consider a FOPTD system whose transfer function is

$$
G(s)=\frac{0.5 e^{-2 s}}{5 s+1} .
$$

By using the proposed tuning table a robustness of $M_{T_{r}}>0.2$ is obtained, and each of the controllers has different levels of control action variations due to changes of $\delta$ in the sampled signal. A measurement gaussian noise is assumed, whose effect is mostly avoided by the choice of the SSOD thresholds $\delta=0.1$.

For transfer function (23) the ratio $L / \tau$ is 0.4 . Searching in Table C.3 the corresponding row, 4 controllers with different values of $\bar{C}(\infty)$ can be found. Each controller is denoted as $C_{i}$ where $i$ is the position of the controller when reading the table from left to right. The parameters of the different controllers are expressed in a dimensionless way, thus, these values must be converted to dimensional parameters using the expressions on equation (13).

Figure 19 shows the open-loop transfer function for all the cases with their respective Tsypkin margin, proving that the robustness requirements have been fulfilled. Additionally, Figure 20 shows the closed-loop response to reference and disturbance step changes on $t=1 \mathrm{~s}$ and $t=25 \mathrm{~s}$ respectively for all the cases. These systems do not present limit cycle oscillations, as expected. The controllers with higher values of $C(\infty)$, which have lower values of IAE, result in faster responses but paying the price of having more abrupt changes in the control action (as shown in Figure 21), as a result of higher derivative filter coefficients. This is more clear observing the response of the system controlled with $C_{1}$, which is a PI controller, with regard to the others, which are PID.

The example shows that it is not reasonable to choose an excessively high value of $\bar{C}(\infty)$, because the improvement in the disturbance IAE is small for a high increase in control action bump. For example, controllers $C_{3}$ and $C_{4}$ 

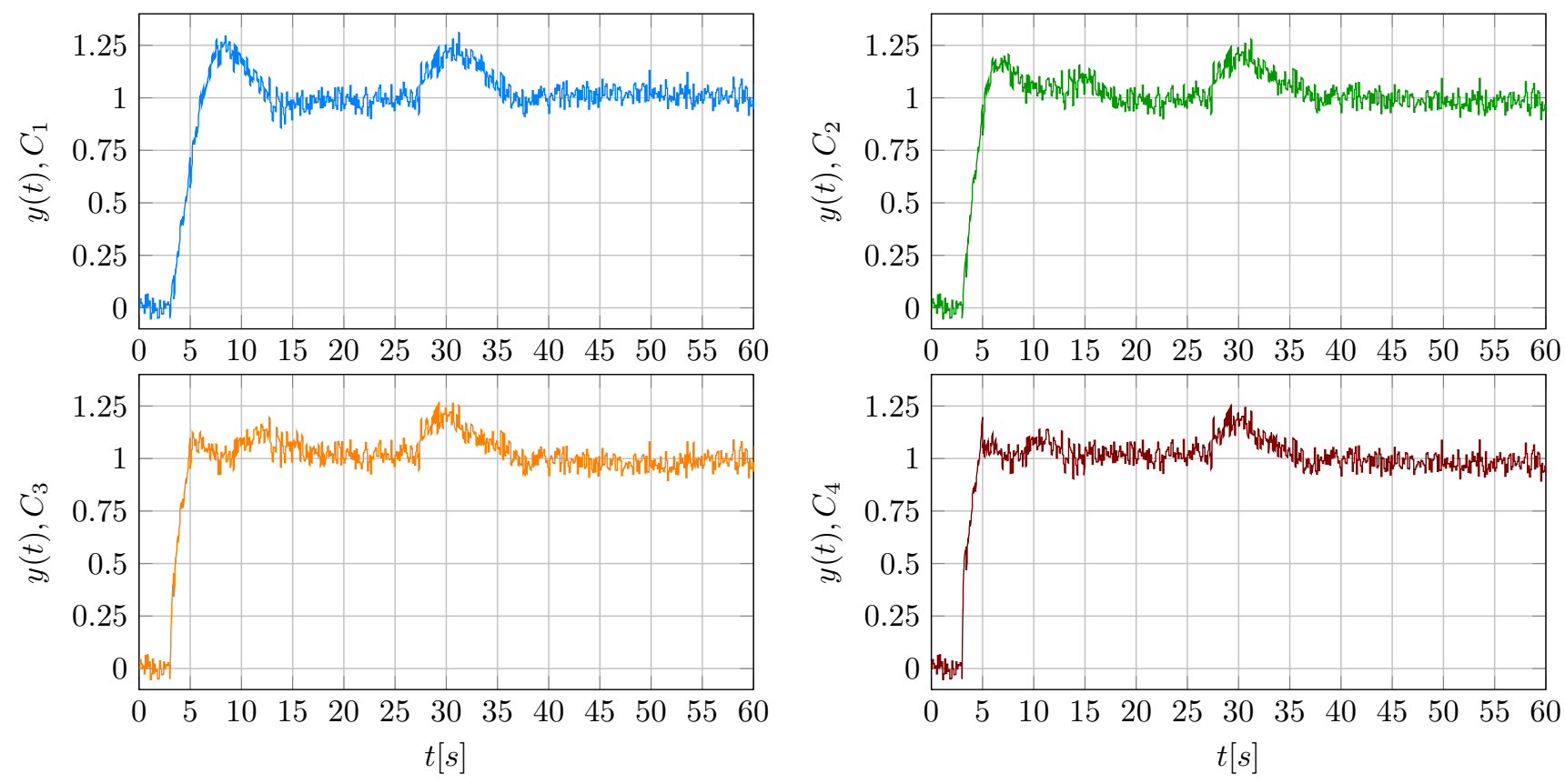

Figure 20: Disturbance response for the considered system with all the controllers.
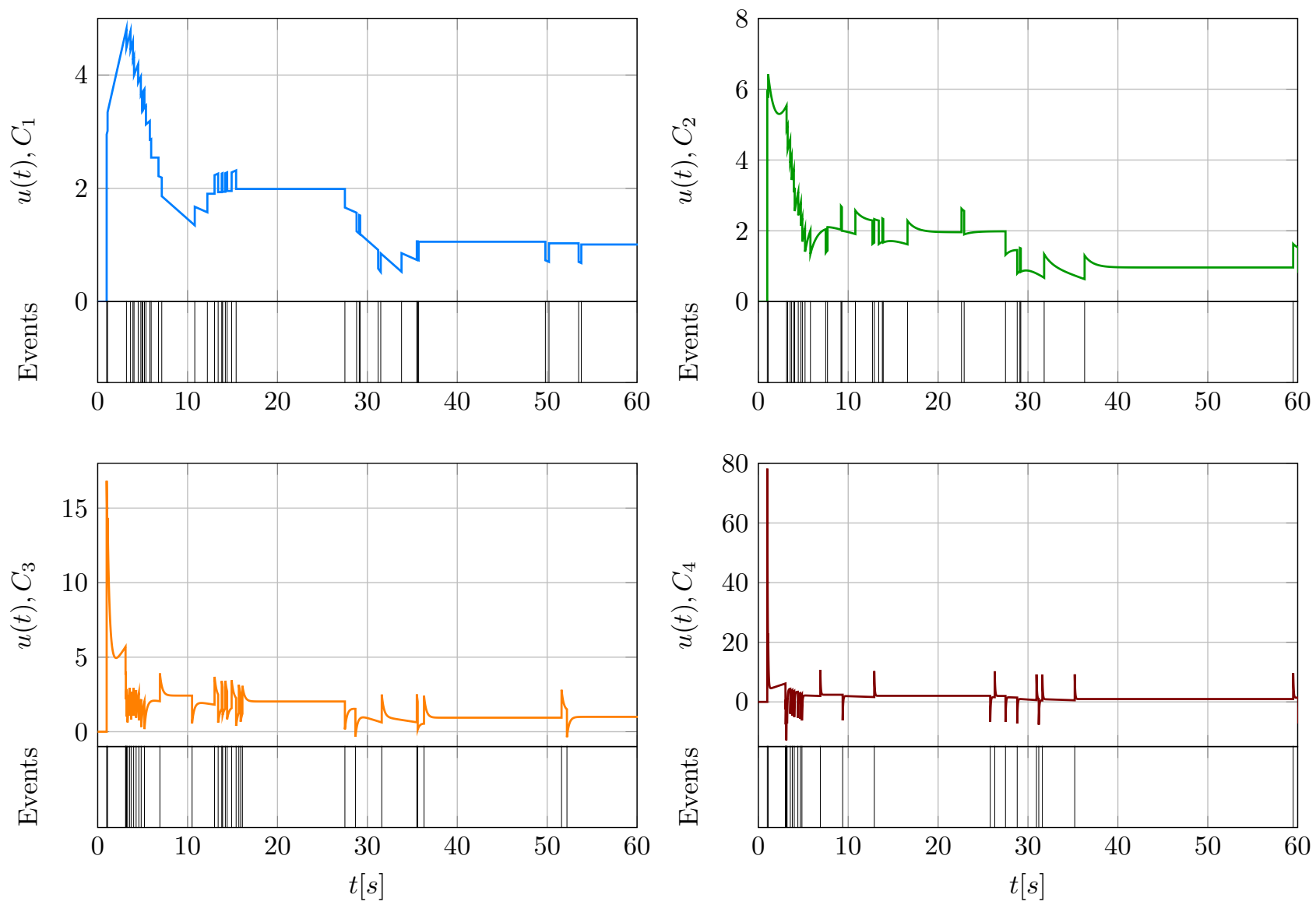

Figure 21: Control action and events generated by crossing the SSOD levels in black for the considered system with all controllers. 
have a very similar temporal response (IAE) but the changes in control action produced by $C_{4}$ are significantly higher than those produced by the controller $C_{3}$. The value of $\delta_{u}$ for $C_{3}$ is $\delta_{u}=K_{p}(1+N) \delta=3.74(1+4) 0.1=1.87$, and $\delta_{u}=8.694$ for $C_{4}$, resulting in a more aggressive control action.

\section{Effect of the FOPTD approximation}

The tuning procedure presented in the previous sections is based on using a FOPTD model. However, the behavior of many actual industrial processes do not correspond strictly to this kind of models. In this sense, it is important to evaluate the effect of this approximation on the final results with the original system, especially on $M_{T}$ since its value determines the presence of limit cycle oscillations. To shed light about this issue, we have considered the following batch of 95 models widely used to evaluate the performance of PID controllers, which represent most of the common dynamics in real applications, [2]:

$$
\begin{aligned}
& G(s)=\frac{e^{-s}}{(T s+1)^{2}}, \\
& T=0.3,0.5,0.7,1,1.3,1.5,2,4,6,8,10,20,50,100,200,500 \\
& G(s)=\frac{1}{(s+1)(T s+1)^{2}}, \\
& T=0.05,0.1,0.2,0.5,2,5,10 \\
& G(s)=\frac{1}{(s+1)^{n}}, \\
& n=3,4,5,6,7,8 \\
& G(s)=\frac{1}{(s+1)(\alpha s+1)\left(\alpha^{2} s+1\right)\left(\alpha^{3} s+1\right)}, \\
& \alpha=0.1,0.2,0.3,0.4,0.5,0.6,0.7,0.8,0.9 \\
& G(s)=\frac{T e^{-L_{1} s}}{\left(T_{1} s+1\right)(T s+1)}, \quad T_{1}+L_{1}=1, \\
& T=1,2,5,10, \quad L_{1}=0.01,0.02,0.05,0.1,0.3,0.5,0.7,0.9,1 \\
& G(s)=\frac{1-\alpha s}{(s+1)^{3}}, \\
& \alpha=0.1,0.2,0.3,0.4,0.5,0.6,0.7,0.8,0.9,1,1.1 \\
& G(s)=\frac{1}{(s+1)\left((s T)^{2}+1.4 s T+1\right)}, \\
& T=0.1,0.2,0.3,0.4,0.5,0.6,0.7,0.8,0.9,1
\end{aligned}
$$

Controllers with $M_{T} \geq 0.1$ and without constraint on $C(\infty)$ have been obtained for all these models using a FOPTD approximation. The values of $M_{T}$ calculated with both the FOPTD and the original transfer functions are presented in Figure 22. It can be seen that the final $M_{T}$ of the original system with the controller calculated for its FOPTD approximation is greater than the $M_{T}$ of the FOPTD approximation. Therefore, the robustness to limit cycle is assured when using controllers designed with the procedure presented in this paper.

\section{Multi-level oscillations}

In the previous sections, single leveled oscillations with $m=1$ have been characterized using the robustness measure $M_{T}$ and a tuning method has been proposed to avoid this kind of limit cycles. Nevertheless, the question if avoiding oscillations with $m=1$ is enough to ensure robustness to oscillations with $m>1$ has not been addressed yet. In this sense, some results were presented in [14] based on the shape of the describing function of the SSOD sampler, however, as commented earlier, the filtering hypothesis that this technique assumes as true is not fulfilled 

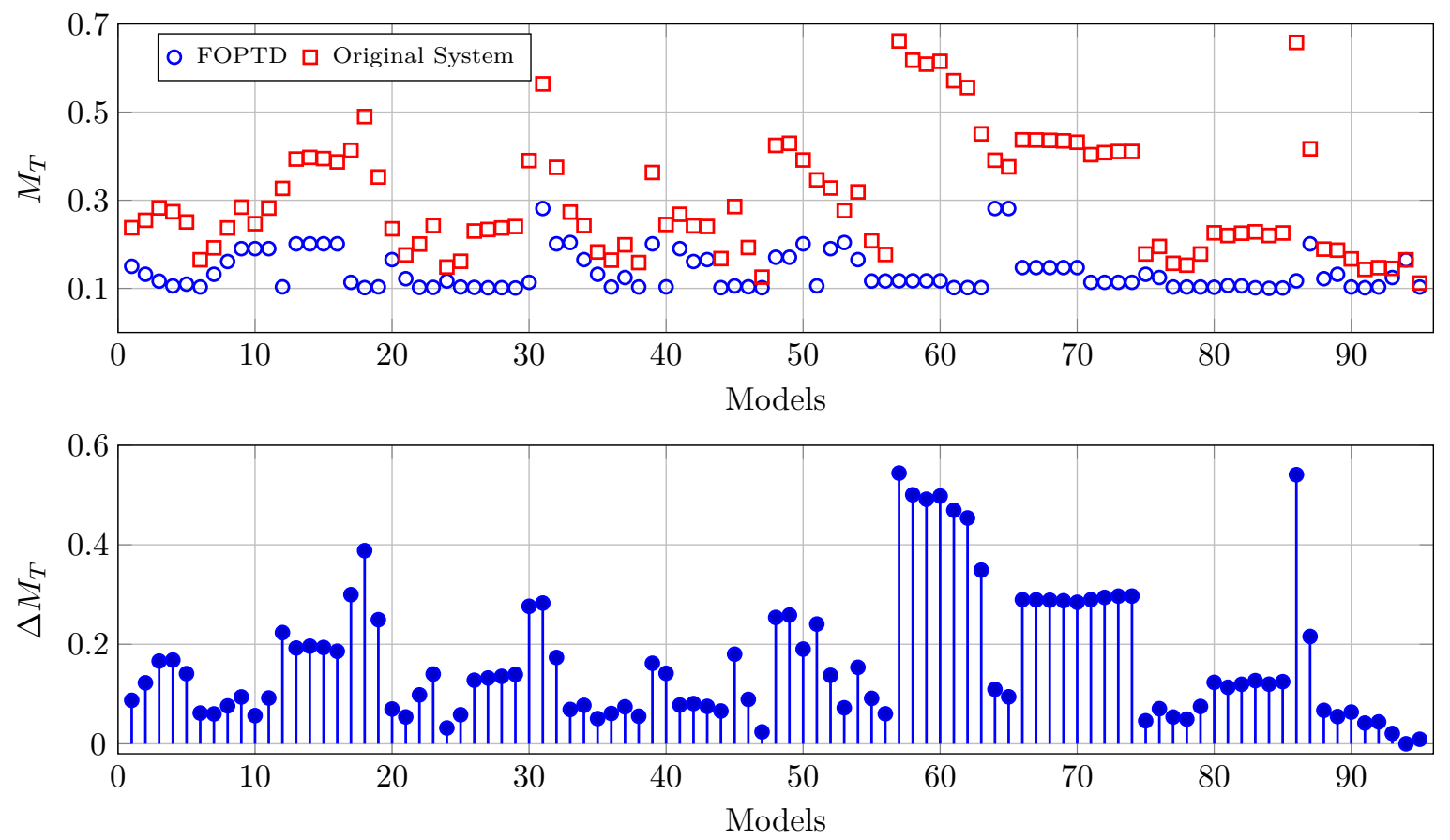

Figure 22: $M_{T}$ and $\Delta M_{T}$ for the considered systems.

for FOPTD systems. Thus, the study of multi-level oscillation $(m>1)$ must follow the same ideas as that for $m=1$, which was presented in section 3 .

As commented in section 3, the complexity of the calculation based on Tsypkin's conditions for oscillation raises significantly with $m$. As an example, let us consider the case $m=2$, whose oscillation conditions derived from equation (6) are:

$$
E C_{p \mid m=2} \mid e\left(\rho_{p} \frac{T_{0}}{2}\right)= \begin{cases}\delta & \text { for } p=1 \\ 2 \delta & \text { for } p=2 \\ \delta & \text { for } p=3 \\ 0 & \text { for } p=4\end{cases}
$$

Evaluating equation (5) for $m=2$ it can be obtained that:

$$
\begin{array}{r}
e\left(\rho_{p} \frac{T_{0}}{2}\right)=-\frac{4 \delta}{\pi} \sum_{n_{\text {odd }}}^{\infty} \frac{1}{n}\left(\Re \{ G _ { \text { ol } } ( n \omega _ { 0 } ) \} \left(\sin \left(\frac{n \pi}{2}\left(\rho_{2}-\rho_{1}\right)\right) \cos \left(n \pi\left(\frac{\rho_{2}+\rho_{1}}{2}-\rho_{p}\right)\right)\right.\right. \\
+2 \sin \left(\frac{n \pi}{2}\left(\rho_{3}-\rho_{2}\right)\right) \cos \left(n \pi\left(\frac{\rho_{3}+\rho_{2}}{2}-\rho_{p}\right)\right) \\
\left.\left.+\sin \left(\frac{n \pi}{2}\left(\rho_{4}-\rho_{3}\right)\right) \cos \left(n \pi\left(\frac{\rho_{4}+\rho_{3}}{2}-\rho_{p}\right)\right)\right)\right) \\
+\frac{4 \delta}{\pi} \sum_{n_{\text {odd }}}^{\infty} \frac{1}{n}\left(\Im \{ G _ { \text { ol } } ( n \omega _ { 0 } ) \} \left(\sin \left(\frac{n \pi}{2}\left(\rho_{2}-\rho_{1}\right)\right) \sin \left(n \pi\left(\frac{\rho_{2}+\rho_{1}}{2}-\rho_{p}\right)\right)\right.\right. \\
+2 \sin \left(\frac{n \pi}{2}\left(\rho_{3}-\rho_{2}\right)\right) \sin \left(n \pi\left(\frac{\rho_{3}+\rho_{2}}{2}-\rho_{p}\right)\right) \\
\left.\left.+\sin \left(\frac{n \pi}{2}\left(\rho_{4}-\rho_{3}\right)\right) \sin \left(n \pi\left(\frac{\rho_{4}+\rho_{3}}{2}-\rho_{p}\right)\right)\right)\right)
\end{array}
$$


Using the equation (26) and the constraints presented in (25), four oscillation conditions are obtained, equation (27). In order to facilitate the lecture of these equations, we have denoted as $C_{r e_{p}}$ and $C_{i m_{p}}$ the coefficients that multiply the real and imaginary parts of $G_{o l}$ respectively for each value of $p$. These coefficients only depend on the values of $\rho_{p}$.

$$
\begin{aligned}
& \sum_{n_{\text {odd }}}^{\infty}\left(\frac{1}{n}\left(\Im\left\{G_{o l}\left(n \omega_{0}\right)\right\} C_{i m_{1}}-\Re\left\{G_{o l}\left(n \omega_{0}\right)\right\} C_{r e_{1}}\right)\right)-\frac{\pi}{4}=0 \\
& \sum_{n_{\text {odd }}}^{\infty}\left(\frac{1}{n}\left(\Im\left\{G_{o l}\left(n \omega_{0}\right)\right\} C_{i m_{2}}-\Re\left\{G_{o l}\left(n \omega_{0}\right)\right\} C_{r e_{2}}\right)\right)-\frac{\pi}{2}=0 \\
& \sum_{n_{\text {odd }}}^{\infty}\left(\frac{1}{n}\left(\Im\left\{G_{o l}\left(n \omega_{0}\right)\right\} C_{i m_{3}}-\Re\left\{G_{o l}\left(n \omega_{0}\right)\right\} C_{r e_{3}}\right)\right)-\frac{\pi}{4}=0 \\
& \sum_{n_{\text {odd }}}^{\infty}\left(\frac{1}{n}\left(\Im\left\{G_{o l}\left(n \omega_{0}\right)\right\} C_{i m_{4}}-\Re\left\{G_{o l}\left(n \omega_{0}\right)\right\} C_{r e_{4}}\right)\right)=0
\end{aligned}
$$

From equation (27) it can be seen that, unlike the case of conditions for $m=1$ given by equations (8), it is impossible to isolate the terms $\Re\left\{G_{o l}\left(j \omega_{o}\right)\right\}$ and $\Im\left\{G_{o l}\left(j \omega_{o}\right)\right\}$, thus a robustness measure as $M_{T}$ with direct visual representation in the Nyquist plane can not be obtained when $m>1$. Then, in order to compare the robustness to oscillations with different $m$ we need to define a more general robustness measure. To this aim, in the case of equation (27), we can use the minimal Euclidean distance between the origin $(0,0,0,0)$ and the points with coordinates given by the left hand members when evaluated in $\left.\rho_{p} \in\right] 0,1\left[, p=1,2,3,4\right.$ and $\omega \in\left[\frac{\omega_{c g}}{200}, \omega_{c g}\right]$. Denoting $R_{2}$ the minimal Euclidean distance for $m=2$, and $L_{p}$ the left hand members of equation (27) for each $p$, the expression for the computation of $R_{2}$ is:

$$
R_{2}=\min _{\omega, \rho_{1}, \cdots, \rho_{4}} \sqrt{\sum_{p=1}^{4} L_{p}\left(\omega, \rho_{1}, \rho_{2}, \rho_{3}, \rho_{4}\right)^{2}}
$$

Taking into account that for any value of $m$ the conditions in (3) can be written as a system of equations similar to (27) but with $2 m$ equations, the general expression for $R_{m}$ is as follows:

$$
R_{m}=\min _{\omega, \rho_{1}, \cdots, \rho_{2 m}} \sqrt{\sum_{p=1}^{2 m} L_{p}\left(\omega, \rho_{1}, \cdots, \rho_{2 m}\right)^{2}}
$$

When $R_{m}=0$, the oscillation requirements are fulfilled, and thus, oscillations with $m$ levels could appear. On the other hand, if $R_{m}>0, m$-leveled oscillation will not occur. Obviously, the complexity of the calculus and the computation time needed to obtain $R_{m}$ increases with the number of oscillation levels $m$ because more combinations of the time fractions $\rho_{p}$ appear than in the case of $m=1$.

Using $R_{m}$ it is possible to compare the robustness of a system to different m-leveled oscillations. With this aim, the cases of $R_{1}, R_{2}$ and $R_{3}$ have been obtained for the batch of 95 models presented in equation (24) with its respective controllers. These robustness measures have been represented in Figure 23. As can be seen, for all these models the value of $R_{m}$ increases with $m$. This shows that in general, by avoiding oscillations for $m=1$ with the proposed tuning method, the system will not present steady state oscillations for higher values of $m$.

\section{Conclusions}

The paper presents a PID tuning procedure for FOPTD systems with Symmetric Send on Delta sampling, that allows to minimize the disturbance IAE while fulfilling constraints on the robustness to oscillations and on the control action changes.

The robustness to avoid limit cycle oscillations due to the SSOD sampling has been taken into account by defining a new robustness measure, $M_{T}$, based on Tsypkin method, that does not require the system to filter out the high order harmonics (as it is required by methods based on the describing function). Therefore, the proposed approach is valid for systems with insufficient filtering characteristics (where the describing function can not be applied).

A set of precomputed controllers has been obtained for dimensionless FOPTD models with different values of $L / \tau$, using a grid on phase margin and on derivative filter parameter $N$. For each controller in the set, the value 


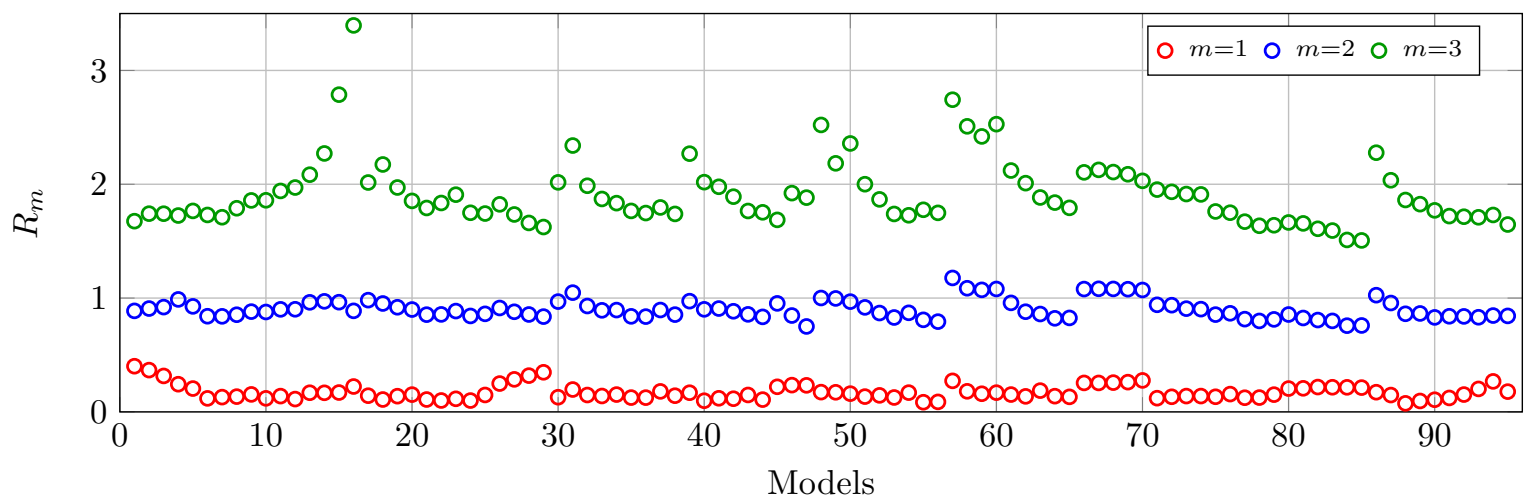

Figure 23: $R_{1}, R_{2}$ and $R_{3}$ for the batch of models with their respective controller obtained in the previous section.

of $M_{T}$ and $C(\infty)$ has been computed. The tuning procedure consists on selecting from this set the controller that minimizes the $I A E$ and fulfills the required $M_{T}$ and $C(\infty)$ constraints.

In order to simplify the application of the tuning procedure, a Java application has been developed (freely available at https://sites.google.com/a/uji.es/freepidtools/ssodTsypkinPid), that computes the controller parameters for the required $M_{T}$ and $C(\infty)$. If the system model is not FOPTD, the applicacion first computes a FOPTD approximation, and calculates de PID controller using that approximated model. Alternatively to this application, a table for tuning controllers with $M_{T}>0.2$ and restrictions on $C(\infty)$ is also provided.

An extensive study with a batch of models has shown that when the system is not FOPTD, the controller obtained with the FOPTD approximation results in higher values of $M_{T}$ than initially required, therefore, the procedure guarantees the robustness to limit cycle oscillations for any system model.

The tuning procedure shows that the derivative filter coefficient, $N$ is a crucial tuning parameter for SSOD PID, because it allows to reach a reasonable compromise between performance (in terms of $I A E$ for example) and control action bumps due to the SSOD sampling.

\section{ACKNOWLEDGMENTS}

This work has been supported by MICINN project number TEC2015-69155-R from the Spanish government, research project 18I411-Uji-b2018-39 from Universitat Jaume I and by CEICE grant number ACIF/2018/244.

\section{References}

[1] Karl J. Åström and Tore Hägglund. Revisiting the Ziegler-Nichols step response method for PID control. Journal of Process Control, 14(6):635-650, sep 2004.

[2] Karl J. Åström and Tore Hägglund. Advanced PID Control. ISA-The Instrumentation, Systems, and Automation Society, 2006.

[3] Pedro Balaguer. Application of dimensional analysis in systems modeling and control design. The Institution of Engineering and Technology, 2013.

[4] Manuel Beschi. Tuning of symmetric send-on-delta proportional-integral controllers. IET Control Theory $\mathcal{E}$ Applications, 8:248-259(11), March 2014.

[5] Manuel Beschi, Sebastián Dormido, José Sánchez, and Antonio Visioli. Characterization of symmetric sendon-delta PI controllers. Journal of Process Control, 22(10):1930-1945, December 2012.

[6] Jesús Chacón, José Sánchez, Antonio Visioli, Luis Yebra, and Sebastián Dormido. Characterization of limit cycles for self-regulating and integral processes with PI control and send-on-delta sampling. Journal of Process Control, 23(6):826-838, 2013. 
[7] G. H. Cohen and G. A. Coon. Theoretical consideration of retarded control. Transactions of the ASME, (75):827-834, 1953.

[8] Sebastián Dormido, Jose Sánchez, and Ernesto Kofman. Muestreo, control y comunicación basados en eventos. Revista Iberoamericana de Automática e Informática Industrial RIAI, 5(1):5 - 26, 2008.

[9] Laura Marie Feeney and Martin Nilsson. Investigating the energy consumption of a wireless network interface in an ad hoc networking environment. In Proceedings IEEE INFOCOM 2001. Conference on Computer Communications. Twentieth Annual Joint Conference of the IEEE Computer and Communications Society (Cat. No. 01CH37213), volume 3, pages $1548-1557$ vol.3, 2001.

[10] S.F. Graebe and A.J. Isaksson. Derivative filter is an integral part of PID design. IEE Proceedings - Control Theory and Applications, 149(1):41-45, 2002.

[11] Birgitta Kristiansson and Bengt Lennartson. Robust Tuning of PI and PID Controllers. Using derivative action despite the sensor noise. IEEE Control Systems Magazine, (February):55-68, 2006.

[12] Oscar Miguel-Escrig, Julio-Ariel Romero-Pérez, and Roberto Sanchis-Llopis. New robustness measure for a kind of event-based PID. IFAC-PapersOnLine, 51(4):781-786, 2018.

[13] Marek Miskowicz. Send-on-delta concept: An event-based data reporting strategy. Sensors, 6(1):49-63, 2006.

[14] Julio Ariel Romero Pérez and Roberto Sanchis Llopis. A new method for tuning PI controllers with symmetric send-on-delta sampling strategy. ISA Transactions, 64:161 - 173, 2016.

[15] Joerns Ploennigs, Volodymyr Vasyutynskyy, and Klaus Kabitzsch. Comparative study of energy-efficient sampling approaches for wireless control networks. Industrial Informatics, IEEE Transactions on, 6(3):416 -424 , aug. 2010.

[16] Julio Ariel Romero, Roberto Sanchis, and Ignacio Penarrocha. A simple rule for tuning event-based PID controllers with symmetric send-on-delta sampling strategy. In Proceedings of the 2014 IEEE Emerging Technology and Factory Automation (ETFA), pages 1-8, Sept 2014.

[17] Julio Ariel Romero-Pérez and Roberto Sanchis Llopis. Tuning and robustness analysis of event-based PID controllers under different event generation strategies. International Journal of Control, pages 1-38, 2017.

[18] Ángel Ruiz, Manuel Beschi, Antonio Visioli, Sebastián Dormido, and Jorge E. Jiménez. A unified event-based control approach for FOPTD and IPTD processes based on the filtered Smith predictor. Journal of the Franklin Institute, 354(2):1239-1264, 2017.

[19] Roberto Sanchis, Julio A. Romero, and Pedro Balaguer. Tuning of PID controllers based on simplified single parameter optimisation. International Journal of Control, 83(9):1785-1798, 2010.

[20] Yakov Z. Tsypkin. Relay control systems. Cambridge University Press, 1984.

[21] John G. Ziegler and Nathaniel B. Nichols. Optimum settings for automatic controllers. trans. ASME, 64(11), 1942.

\section{Appendix A. Tsypkin method calculations}

Consider that the sampled error signal $\bar{e}(t)$ follows a ladder-type form as shown in Figure 5, then the expression of $\bar{e}(t)$ can be obtained through Fourier series expansion:

$$
\bar{e}(t)=\frac{a_{0}}{2}+\sum_{n=1}^{\infty}\left(a_{n} \cos \left(\omega_{n} t\right)+b_{n} \sin \left(\omega_{n} t\right)\right)
$$

As the error signal is supposed centered around 0 and symmetric the coefficient $a_{0}$ is equal to 0 . For the other coefficients: 


$$
\begin{aligned}
a_{n}= & \frac{2}{T_{o}} \int_{\rho_{1} \frac{T_{o}}{2}}^{\rho_{2} \frac{T_{o}}{2}} \delta \cos \left(\omega_{n} t\right) d t+\frac{2}{T_{o}} \int_{\rho_{2} \frac{T_{o}}{2}}^{\rho_{3} \frac{T_{o}}{2}} 2 \delta \cos \left(\omega_{n} t\right) d t+\ldots+\frac{2}{T_{o}} \int_{\rho_{m} \frac{T_{o}}{2}}^{\rho_{m+1} \frac{T_{o}}{2}} m \delta \cos \left(\omega_{n} t\right) d t+ \\
& +\frac{2}{T_{o}} \int_{\rho_{m+1} \frac{T_{o}}{2}}^{\rho_{m+2} \frac{T_{o}}{2}}(m-1) \delta \cos \left(\omega_{n} t\right) d t+\ldots+\frac{2}{T_{0}} \int_{\rho_{2 m-1} \frac{T_{0}}{2}}^{\rho_{2 m} \frac{T_{o}}{2}} \delta \cos \left(\omega_{n} t\right) d t+ \\
& +\frac{2}{T_{o}} \int_{\frac{T_{o}}{2}+\rho_{1} \frac{T_{o}}{2}}^{\frac{T_{o}}{2}+\rho_{2} \frac{T_{o}}{2}}-\delta \cos \left(\omega_{n} t\right) d t+\ldots+\frac{2}{T_{o}} \int_{\frac{T_{o}}{2}+\rho_{m} \frac{T_{o}}{2}}^{\frac{T_{o}}{2}+\rho_{m+1} \frac{T_{o}}{2}}-m \delta \cos \left(\omega_{n} t\right) d t+ \\
& +\frac{2}{T_{o}} \int_{\frac{T_{o}}{2}+\rho_{m+1} \frac{T_{o}}{2}}^{\frac{T_{o}}{2}+\rho_{m+2} \frac{T_{o}}{2}}-(m-1) \delta \cos \left(\omega_{n} t\right) d t+\ldots+\frac{2}{T_{o}} \int_{\frac{T_{o}}{2}+\rho_{2 m-1} \frac{T_{o}}{2}}^{\frac{T_{o}}{2}}-\delta \cos \left(\omega_{n} t\right) d t
\end{aligned}
$$

Solving and arranging the equation, the first Fourier coefficient is obtained:

$$
a_{n}=\left\{\begin{array}{cl}
0 & \text { if } \mathrm{n} \text { is even } \\
\frac{2 \delta}{n \pi}\left(\sum_{i=1}^{m} i\left(\sin \left(n \pi \rho_{i+1}\right)-\sin \left(n \pi \rho_{i}\right)\right)+\sum_{i=m+1}^{2 m-1}(2 m-i)\left(\sin \left(n \pi \rho_{i+1}\right)-\sin \left(n \pi \rho_{i}\right)\right)\right) & \text { if } n \text { is odd }
\end{array}\right.
$$

In a similar way, the second coefficient is obtained:

$$
b_{n}=\left\{\begin{array}{cl}
\frac{2 \delta}{n \pi}\left(\sum_{i=1}^{m} i\left(\cos \left(n \pi \rho_{i}\right)-\cos \left(n \pi \rho_{i+1}\right)\right)+\sum_{i=m+1}^{2 m-1}(2 m-i)\left(\cos \left(n \pi \rho_{i}\right)-\cos \left(n \pi \rho_{i+1}\right)\right)\right) & \text { if } n \text { is even } n \text { is odd }
\end{array}\right.
$$

Substituting $a_{0}, a_{n}$ and $b_{n}$ in equation (A.1), $\bar{e}(t)$ is obtained. Taking into account that $e=-L \bar{e}$, and knowing that $L$ is the open-loop transfer function of the system $\left(L=G_{o l}(j \omega)\right)$, the general expression for $e(t)$ results in:

$$
\begin{aligned}
e\left(\rho_{p} \frac{T_{o}}{2}\right)=-\frac{4 \delta}{\pi} \sum_{n_{\text {odd }}}^{\infty} \frac{1}{n}\left(\Re \{ G _ { \text { ol } } ( j n \omega _ { o } ) \} \left(\sum_{i=1}^{m} i \sin \left(\frac{n \pi}{2}\left(\rho_{i+1}-\rho_{i}\right)\right) \cos \left(n \pi\left(\frac{\rho_{i+1}+\rho_{i}}{2}-\rho_{p}\right)\right)\right.\right. \\
\left.\left.+\sum_{i=m+1}^{2 m-1}(2 m-i) \sin \left(\frac{n \pi}{2}\left(\rho_{i+1}-\rho_{i}\right)\right) \cos \left(n \pi\left(\frac{\rho_{i+1}+\rho_{i}}{2}-\rho_{p}\right)\right)\right)\right) \\
-\frac{4 \delta}{\pi} \sum_{n_{\text {odd }}}^{\infty} \frac{1}{n}\left(\Im \{ G _ { \text { ol } } ( j n \omega _ { o } ) \} \left(\sum_{i=1}^{m} i \sin \left(\frac{n \pi}{2}\left(\rho_{i+1}-\rho_{i}\right)\right) \sin \left(n \pi\left(\frac{\rho_{i+1}+\rho_{i}}{2}-\rho_{p}\right)\right)\right.\right. \\
\left.\left.+\sum_{i=m+1}^{2 m-1}(2 m-i) \sin \left(\frac{n \pi}{2}\left(\rho_{i+1}-\rho_{i}\right)\right) \sin \left(n \pi\left(\frac{\rho_{i+1}+\rho_{i}}{2}-\rho_{p}\right)\right)\right)\right)
\end{aligned}
$$

\section{Appendix B. Dimensionless Indexes calculations}

Considering a SISO closed loop with input disturbance, the relation between the dimensionless error $\bar{E}(\bar{s})$ and the load disturbance $\bar{P}(\bar{s})$ is:

$$
\frac{\bar{E}(\bar{s})}{\bar{P}(\bar{s})}=\frac{\bar{G}(\bar{s})}{1+\bar{G}(\bar{s}) \bar{C}(\bar{s})}
$$


which substituting with the given expressions for $\bar{G}(\bar{s})$ and $\bar{C}(\bar{s})$ :

$$
\frac{\bar{E}(\bar{s})}{\bar{P}(\bar{s})}=\frac{e^{-\bar{s}}}{\left[\frac{\tau}{L} \bar{s}+1+\phi_{1}\left(1+\frac{1}{\phi_{2} \bar{s}}+\frac{N \phi_{3} \bar{s}}{N+\phi_{3} \bar{s}}\right) e^{-\bar{s}}\right]}
$$

similarly, for a system with dimensions:

$$
\frac{E(s)}{P(s)}=\frac{K e^{-s L}}{\left[\tau s+1+\phi_{1}\left(1+\frac{1}{L \phi_{2} s}+\frac{N L \phi_{3} s}{N+L \phi_{3} s}\right) e^{-s L}\right]}
$$

then, the relationship between those two expressions is:

$$
\frac{E(s)}{P(s)}=K \frac{\bar{E}(\bar{s}=s L)}{\bar{P}(\bar{s}=s L)}
$$

From this expression it can be obtained the effect of a step disturbance of magnitude 1 on $P(s)$ from a dimensionless step of magnitude 1 on $\bar{P}(\bar{s})$ :

$$
E(s)=K \frac{\bar{E}(s L)}{\bar{P}(s L)} P(s)=K \frac{\bar{E}(s L)}{\frac{1}{s L}} \frac{1}{s}=K L \bar{E}(s L)
$$

applying over this expression the linearity property of the Laplace transform, the gain $K$ can be extracted. Then, applying the change of scale of the Laplace transform for dealing with the delay:

$$
\mathscr{L}^{-1}\{E(s)\}=K \bar{e}(t / L)
$$

where

$$
\bar{e}(t):=\mathscr{L}^{-1}\{\bar{E}(\bar{s})\}
$$

Being $\overline{I A E}$ the $I A E$ obtained for the dimensionless model:

$$
\overline{I A E}:=\int_{0}^{\infty}|\bar{e}(t)| d t
$$

Using the expression (B.1) to calculate the index for a generic system:

$$
I A E=\int_{0}^{\infty}|K \bar{e}(t / L)| d t
$$

Extracting the gain from the integral and performing a variable change $t^{\prime}=t / L$ for solving the integral:

$$
I A E=|K| L \overline{I A E}
$$

\section{Appendix C. Tuning table}

See Table C.3. 


\begin{tabular}{|c|c|c|c|c|c|c|c|c|c|c|c|c|c|c|c|}
\hline \multirow{2}{*}{$\mathbf{L} / \tau$} & \multicolumn{3}{|c|}{$\overline{\mathbf{C}}(\infty)<\mathbf{1}$} & \multicolumn{3}{|c|}{$\overline{\mathbf{C}}(\infty)<\mathbf{2}$} & \multicolumn{3}{|c|}{$\overline{\mathbf{C}}(\infty)<\mathbf{5}$} & \multicolumn{3}{|c|}{$\overline{\mathbf{C}}(\infty)<10$} & \multicolumn{3}{|c|}{$\forall \overline{\mathbf{C}}(\infty)$} \\
\hline & $\overline{\mathbf{K}_{\mathbf{p}}}$ & $\overline{\mathbf{T}_{\mathrm{i}}}$ & $\mathbf{N}$ & $\overline{\mathbf{K}_{\mathrm{p}}}$ & $\overline{\mathbf{T}_{\mathbf{i}}}$ & $\mathbf{N}$ & $\overline{\mathbf{K}_{\mathbf{p}}}$ & $\overline{T_{i}}$ & $\mathbf{N}$ & $\overline{\mathbf{K}_{\mathbf{p}}}$ & $\overline{\mathbf{T}_{\mathbf{i}}}$ & $\mathbf{N}$ & $\overline{\mathbf{K}_{\mathbf{p}}}$ & $\overline{\mathbf{T}_{\mathbf{i}}}$ & $\mathbf{N}$ \\
\hline 0.1 & & & & & & & 3.97 & 7.54 & & $*$ & * & & $*$ & $*$ & \\
\hline 0.2 & & & & & & & 2.44 & 3.22 & 0.5 & 2.7 & 3.0 & 2.0 & 2.9 & 3.13 & 20.0 \\
\hline 0.3 & & & & 1.97 & 2.97 & & 2.01 & 2.39 & 1.0 & 2.17 & 2.34 & 3.0 & 2.46 & 2.37 & 20.0 \\
\hline 0.4 & & & & 1.64 & 2.34 & & 1.66 & 1.9 & 1.0 & 1.87 & 2.0 & 4.0 & 2.07 & 2.05 & 20.0 \\
\hline 0.5 & & & & 1.41 & 2.01 & & 1.55 & 1.65 & 2.0 & 1.68 & 1.69 & 4.0 & 1.78 & 1.79 & 12.0 \\
\hline 0.6 & & & & 1.15 & 1.38 & 0.5 & 1.36 & 1.43 & 2.0 & 1.45 & 1.59 & 5.0 & 1.48 & 1.6 & 6.0 \\
\hline 0.7 & & & & 1.06 & 1.28 & 0.5 & 1.24 & 1.4 & 2.0 & 1.35 & 1.51 & 5.0 & $*$ & $*$ & $*$ \\
\hline 0.8 & 1.0 & 1.9 & & 0.96 & 1.18 & 0.5 & 1.14 & 1.31 & 2.0 & 1.25 & 1.47 & 7.0 & 1.26 & 1.48 & 8.0 \\
\hline 0.9 & 0.94 & 1.36 & & 0.95 & 1.12 & 1.0 & 1.08 & 1.28 & 3.0 & $*$ & $*$ & $*$ & $*$ & $*$ & $*$ \\
\hline 1.0 & 0.9 & 1.35 & & 0.91 & 1.07 & 1.0 & 0.98 & 1.2 & 2.0 & 1.05 & 1.29 & 4.0 & 1.1 & 1.37 & 12.0 \\
\hline 1.1 & 0.83 & 1.23 & & 0.85 & 1.05 & 1.0 & 0.99 & 1.22 & 4.0 & $*$ & $*$ & $*$ & $*$ & $*$ & $*$ \\
\hline 1.2 & 0.79 & 1.22 & & 0.81 & 1.0 & 1.0 & 0.89 & 1.09 & 2.0 & * & * & * & * & $*$ & $*$ \\
\hline 1.3 & 0.74 & 1.13 & & 0.77 & 0.96 & 1.0 & 0.87 & 1.1 & 3.0 & $*$ & $*$ & $*$ & $*$ & $*$ & $*$ \\
\hline 1.4 & 0.72 & 1.13 & & 0.74 & 0.95 & 1.0 & 0.84 & 1.06 & 3.0 & * & * & * & * & $*$ & $*$ \\
\hline 1.5 & 0.65 & 0.87 & 0.5 & 0.71 & 0.91 & 1.0 & 0.83 & 1.07 & 5.0 & $*$ & * & $*$ & $*$ & $*$ & $*$ \\
\hline 1.6 & 0.62 & 0.84 & 0.5 & 0.68 & 0.88 & 1.0 & 0.8 & 1.04 & 5.0 & $*$ & * & $*$ & * & $*$ & $*$ \\
\hline 1.7 & 0.61 & 0.81 & 0.5 & 0.67 & 0.86 & 1.0 & 0.78 & 1.01 & 5.0 & $*$ & $*$ & $*$ & * & $*$ & $*$ \\
\hline 1.8 & 0.6 & 0.83 & 0.5 & 0.65 & 0.83 & 1.0 & 0.71 & 0.91 & 2.0 & $*$ & * & * & $*$ & $*$ & $*$ \\
\hline 1.9 & 0.58 & 0.8 & 0.5 & 0.63 & 0.84 & 1.0 & 0.69 & 0.88 & 2.0 & $*$ & $*$ & $*$ & $*$ & $*$ & $*$ \\
\hline 2.0 & 0.57 & 0.78 & 0.5 & 0.62 & 0.82 & 1.0 & 0.71 & 0.93 & 4.0 & $*$ & $*$ & $*$ & $*$ & $*$ & $*$ \\
\hline 2.1 & 0.56 & 0.76 & 0.5 & 0.66 & 0.84 & 2.0 & 0.7 & 0.9 & 4.0 & $*$ & * & $*$ & $*$ & $*$ & $*$ \\
\hline 2.2 & 0.55 & 0.75 & 0.5 & 0.64 & 0.86 & 2.0 & 0.67 & 0.87 & 3.0 & $*$ & $*$ & $*$ & $*$ & $*$ & $*$ \\
\hline 2.3 & 0.54 & 0.73 & 0.5 & 0.63 & 0.84 & 2.0 & 0.67 & 0.86 & 4.0 & * & * & * & $*$ & $*$ & $*$ \\
\hline 2.4 & 0.53 & 0.72 & 0.5 & 0.62 & 0.82 & 2.0 & 0.65 & 0.84 & 3.0 & $*$ & $*$ & $*$ & $*$ & $*$ & $*$ \\
\hline 2.5 & 0.52 & 0.7 & 0.5 & 0.61 & 0.81 & 2.0 & 0.64 & 0.83 & 3.0 & $*$ & $*$ & $*$ & $*$ & $*$ & $*$ \\
\hline 2.6 & 0.51 & 0.69 & 0.5 & 0.6 & 0.8 & 2.0 & 0.62 & 0.81 & 3.0 & 0.67 & 0.87 & 10.0 & * & $*$ & $*$ \\
\hline 2.7 & 0.5 & 0.67 & 0.5 & 0.6 & 0.78 & 2.0 & 0.62 & 0.8 & 3.0 & 0.66 & 0.86 & 10.0 & $*$ & $*$ & $*$ \\
\hline 2.8 & 0.5 & 0.67 & 0.5 & 0.59 & 0.77 & 2.0 & 0.61 & 0.79 & 3.0 & 0.65 & 0.85 & 10.0 & $*$ & $*$ & $*$ \\
\hline 2.9 & 0.49 & 0.66 & 0.5 & 0.58 & 0.76 & 2.0 & 0.6 & 0.77 & 3.0 & 0.64 & 0.83 & 10.0 & $*$ & $*$ & $*$ \\
\hline 3.0 & 0.49 & 0.65 & 0.5 & 0.58 & 0.75 & 2.0 & 0.59 & 0.76 & 3.0 & 0.63 & 0.83 & 10.0 & $*$ & $*$ & $*$ \\
\hline
\end{tabular}

Table C.3: Tuning table for $M_{T_{r}}>0.2$. (*): Take the parameters for the precedent case of $\bar{C}(\infty)$. 